\title{
Hom-configurations and noncrossing partitions
}

\author{
Raquel Coelho Simões
}

Received: 3 February 2011 / Accepted: 4 July 2011 / Published online: 6 August 2011

(C) Springer Science+Business Media, LLC 2011

\begin{abstract}
We study maximal Hom-free sets in the $\tau$ [2]-orbit category $C(Q)$ of the bounded derived category for the path algebra associated to a Dynkin quiver $Q$, where $\tau$ denotes the Auslander-Reiten translation and [2] denotes the square of the shift functor. We prove that these sets are in bijection with periodic combinatorial configurations, as introduced by Riedtmann, certain Hom $_{\leq 0}$-configurations, studied by Buan, Reiten and Thomas, and noncrossing partitions of the Coxeter group associated to $Q$ which are not contained in any proper standard parabolic subgroup. Note that Reading has proved that these noncrossing partitions are in bijection with positive clusters in the associated cluster algebra. Finally, we give a definition of mutation of maximal Hom-free sets in $\mathcal{C}(Q)$ and prove that the graph of these mutations is connected.
\end{abstract}

Keywords Cluster combinatorics · Derived category · Exceptional sequences · Hom-configurations $\cdot$ Mutations $\cdot$ Noncrossing partitions $\cdot$ Perpendicular categories · Reflection functors · Quiver representations

\section{Introduction}

Let $Q$ be a Dynkin quiver, $\mathcal{D}^{b}(Q)$ the bounded derived category for the path algebra associated to $Q$, with shift functor [1] and Auslander-Reiten translation $\tau$.

Our main object of study is the set of Hom-configurations in the orbit category $\mathcal{C}(Q):=\mathcal{D}^{b}(Q) / \tau[2]$, which is triangulated by Keller [19]. A Hom-configuration is defined to be a maximal Hom-free set of indecomposable objects in this category. We will give bijections between the collection of Hom-configurations in $\mathcal{C}(Q)$ and collections of other representation-theoretic and combinatorial objects.

R. Coelho Simões $(\varangle)$

School of Mathematics, University of Leeds, Leeds LS2 9JT, UK

e-mail: rcsimoes@maths.leeds.ac.uk 
One such collection is the set of combinatorial configurations, which were introduced by Riedtmann [22] in order to classify self-injective algebras of finite representation type. A combinatorial configuration can be regarded as a certain Hom-free collection of indecomposable objects in the bounded derived category of finitely generated modules over a path algebra associated to a Dynkin quiver. Riedtmann proved (cf. [22, 23]) that combinatorial configurations in type $A$ and $D$ are invariant under the functor $\tau[2]$.

Motivated by this, the authors of [8] studied the Ext-version of these configurations in the bounded derived category $\mathcal{D}^{b}$ for any finite-dimensional hereditary algebra, the so called Ext-configurations. The authors proved that these objects are invariant under the functor $\tau^{-1}[1]$. This implies that Ext-configurations in the bounded derived category $\mathcal{D}^{b}$ are in one-to-one correspondence with Ext-configurations in the cluster category $\mathcal{D}^{b} / \tau^{-1}[1]$, which are called cluster-tilting objects. These objects were proved to be in bijection with maximal Ext-free sets in the cluster category (cf. [8, 2.3]). One should then expect that a similar result holds for combinatorial configurations in the orbit category $\mathcal{D}^{b}(Q) / \tau[2]$, where $\mathcal{D}^{b}(Q)$ is the bounded derived category for the path algebra associated to any Dynkin quiver $Q$.

We prove that Hom-configurations in $\mathcal{C}(Q)$ are in bijection with periodic combinatorial configurations, i.e., combinatorial configurations in $\mathcal{D}^{b}(Q)$ which are invariant under $\tau[2]$.

Riedtmann also gave a natural bijection between the set of combinatorial configurations for type $A_{n}$ and the set $N C(n)$ of classical noncrossing partitions of the set $\{1, \ldots, n\}$, which was introduced by Kreweras [20] in 1972.

Later, in the early 2000's, Brady [5] and Bessis [2] independently introduced an algebraic generalization of classical noncrossing partitions. To each finite Coxeter group $W$ these authors associate a poset, called the poset of noncrossing partitions of $W$, which we denote by $N C(W)$. The posets $N C(n)$ and $N C\left(A_{n-1}\right)$ are known to be isomorphic [3].

The initial motivation for this article was to generalize Riedtmann's bijection to any Dynkin case, using Hom-configurations. However, a simple computation in type $D_{4}$ shows that the number of Hom-configurations in $\mathcal{C}(Q)$ is different from the number of noncrossing partitions of type $D_{3}$. So we reinterpret Riedtmann's bijection as follows.

Each finite Coxeter group $W$ has a number associated to it, known as the Catalan number of type $W$. These numbers occur in various counting problems, and in particular, the number of noncrossing partitions associated to $W$ is given by the corresponding Catalan number (cf. [2]).

There is also another number that is associated to $W$, the so called positive Catalan number (see [14]). This number counts a special subset of the noncrossing partitions. This subset, which will be denoted by $\mathrm{NC}^{+}(W)$, consists of the noncrossing partitions which are not contained in any proper standard parabolic subgroup. Reading [21] has proved that this subset is in bijection with positive clusters in the associated cluster algebra ([12], see also [13]). For this reason, we say that these noncrossing partitions are positive.

The Catalan number of type $A_{n-1}$ coincides with the positive Catalan number of type $A_{n}$. Given this fact, it is possible to reinterpret Riedtmann's bijection as a bijection between $A_{n}$ combinatorial configurations and $A_{n}$ positive noncrossing partitions. 
We then give a combinatorial description of positive noncrossing partitions for type $A$, giving an explicit one-to-one correspondence $f$ between $N C\left(A_{n-1}\right)$ and $N C^{+}\left(A_{n}\right)$. We also give a bijection $\varphi$ between positive noncrossing partitions of the Coxeter group $W_{Q}$ associated to any Dynkin quiver $Q$ and Hom-configurations in $\mathcal{C}(Q)$. This bijection generalizes Riedtmann's bijection, in the sense that Riedtmann's bijection is given by the composition of $\varphi$ (in the $A_{n}$ case) with $f$.

Our work is closely related to [10]. In this paper the authors give a natural bijection between $m$-clusters and $m$-noncrossing partitions, for $m \geq 1$, using special classes of exceptional sequences in the bounded derived category. The sets of elements of one of these special classes of exceptional sequences are called $m$-Hom $\leq 0^{-}$ configurations. These configurations are contained in $\mathcal{D}_{\leq m}^{\geq 0}$, the full additive subcategory of the bounded derived category generated by the indecomposable objects of $K Q-\bmod [i]$, with $0 \leq i \leq m$.

Hom-configurations turn out to be in bijection with 1 - Hom $\leq 0$-configurations contained in $D_{\leq 1}^{(\geq 0)-}$, the full additive subcategory of the bounded derived category generated by the indecomposable objects of $K Q-\bmod \cup K Q-\bmod [1]$ other than the projective modules.

The main results presented in this paper can be summarized in the following theorem.

Theorem 1.1 Let $Q$ be a Dynkin quiver and $\mathcal{C}(Q)$ the orbit category $\mathcal{D}^{b}(Q) / \tau[2]$. Then the following collections of objects are in bijection:

(1) Hom-configurations in $\mathcal{C}(Q)$;

(2) Hom $_{\leq 0}$-configurations contained in $D_{\leq 1}^{(\geq 0)-}$;

(3) periodic combinatorial configurations;

(4) sincere Hom-free sets in $K Q$ - mod;

(5) positive noncrossing partitions of the Coxeter group $W_{Q}$ associated to $Q$.

The paper is organized as follows. We first prove that the subcategory of $\mathcal{C}(Q)$

$$
{ }^{\perp} M_{Q}^{\perp}=\left\{X \in \mathcal{C}(Q) \mid \operatorname{Hom}_{\mathcal{C}(Q)}(X, M)=0=\operatorname{Hom}_{\mathcal{C}(Q)}(M, X)\right\},
$$

where $M$ is an indecomposable object of $\mathcal{C}(Q)$, is equivalent to $\mathcal{C}\left(Q^{\prime}\right)$ where $Q^{\prime}$ is a disjoint union of quivers of Dynkin type, whose number of vertices is $n-1$.

This result gives us a strategy to prove some of the results in this paper. This strategy is to use induction and reduce to the simpler case when we have a Homconfiguration which contains a simple projective indecomposable module.

In Sect. 3, we prove the bijection between (1) and (2) in Theorem 1.1. The one-toone correspondence between (1) and (4) is proved in Sect. 4. This result is crucial to prove the relation between Hom-configurations and positive noncrossing partitions.

In Sect. 5 we give a bijection between (1) and (5). We note that Buan, Reiten and Thomas [9, Theorem 7] provide a different Coxeter-theoretic description for the noncrossing partitions in bijection with $\mathrm{Hom}_{\leq 0}$-configurations contained in $\mathcal{D}_{\leq 1}^{(\geq 0)-}$.

In Sect. 6, we prove the bijection between (1) and (3). We use some results in [6] and the fact that the number of Hom-configurations is given by the so called positive Fuss-Catalan number corresponding to the Coxeter group $W_{Q}$ associated 
to the Dynkin quiver $Q$. This fact follows immediately from the bijection between Hom-configurations in $\mathcal{C}(Q)$ and positive noncrossing partitions.

In Sect. 7 we give a combinatorial description of this class of noncrossing partitions for type $A$ and check that the bijection between (1) and (5) generalizes the bijection given by Riedtmann in type $A$.

Finally, in Sect. 8 we give a definition of mutation of Hom-configurations and prove that the graph of these mutations is connected.

\section{Perpendicular category for Hom-configurations-main tool}

Firstly let us fix some notation. Denote by $K$ an algebraically closed field, $Q$ a simply laced Dynkin quiver with $n$ vertices, $Q_{0}$ the set of vertices of $Q, h$ the Coxeter number associated to $Q$ and $K Q$ the path algebra. All modules considered will be left finite-dimensional $K Q$-modules. The support of a module $M$, which we will denote by $\operatorname{supp}(M)$, is the set of vertices $i$ of $Q$ for which $M_{i} \neq 0$. The bounded derived category of $K Q$-modules will be denoted by $\mathcal{D}^{b}(Q)$. We know that the indecomposable objects in $\mathcal{D}^{b}(Q)$ are of the form $M[i]$, for some indecomposable $K Q$-module $M$ and some integer $i$. If $X=M[i]$ is an indecomposable object in $\mathcal{D}^{b}(Q)$, we denote by $\bar{X}$ the corresponding indecomposable $K Q$-module $M$, and by $d(X)$ its degree, i.e., $d(X)=d(M[i])=i$.

We define a partial order in the set ind $\mathcal{D}^{b}(Q)$, the subcategory of isomorphism classes of indecomposable objects in $\mathcal{D}^{b}(Q)$, as follows. Given $X, Y \in$ ind $\mathcal{D}^{b}(Q)$, we say that $X \preceq Y$ if there is a path from $X$ to $Y$ in the Auslander-Reiten quiver of $\mathcal{D}^{b}(Q)$. It is clear that $\preceq$ is indeed a partial order. Fix a refinement $\leq$ of $\preceq$ to a total order that satisfies the following property:

$$
X \leq Y \quad \text { if } d(X)<d(Y)
$$

Let $\mathcal{C}(Q)$ be the category $\mathcal{D}^{b}(Q) / \tau^{h-1}$, where $\tau$ is the Auslander-Reiten translate. Note that $\mathcal{C}(Q)$ can also be defined to be the category $\mathcal{D}^{b}(Q) / \tau$ [2], where [- ] is the shift functor and $\tau$ is the AR-translate in $\mathcal{D}^{b}(Q)$ (cf. [15]).

It is easy to check that the objects in the subcategory

$$
\mathcal{E}(Q)=\operatorname{ind}(K Q-\bmod \cup(K Q-\bmod \backslash \mathcal{I})[1])
$$

of $\mathcal{D}^{b}(Q)$ where $\mathcal{I}$ denotes the set of injective modules, is a fundamental domain for the action of $\tau$ [2] on ind $\mathcal{D}^{b}(Q)$. From now on, we identify the objects in ind $\mathcal{C}(Q)$ with their representatives in this fundamental domain, unless stated otherwise.

The following remark is going to be useful later.

Remark 2.1 It follows very easily from the Serre duality that, for any pair of objects $X, Y$ in $\mathcal{D}^{b}(Q)$, we have $\operatorname{Hom}_{\mathcal{D}^{b}(Q)}(X, \tau Y[2]) \simeq \operatorname{Hom}_{\mathcal{D}^{b}(Q)}(Y[1], X) \simeq$ $\operatorname{Ext}_{\mathcal{D}^{b}(Q)}^{-1}(Y, X)$. 
Proposition 2.2 Let $X, Y \in \mathcal{E}(Q)$.

(1) We have $\operatorname{Hom}_{\mathcal{D}^{b}(Q)}\left(X, \tau^{k} Y[2 k]\right)=0$ for all $k \neq 0,1$.

(2) $\operatorname{Hom}_{\mathcal{D}^{b}(Q)}\left(X, \tau^{k} Y[2 k]\right) \neq 0$ for at most one value of $k$.

Proof (1) Let $k=-1$ and suppose $d(Y)=1$. Note that $d\left(\tau^{-1} Y[-2]\right)=0$ if and only if $Y=I[1]$ for some injective $K Q$-module $I$, which contradicts the hypothesis that $Y \in \mathcal{E}(Q)$. Hence we have $d\left(\tau^{-1} Y[-2]\right)=-1$ and it is clear that $\operatorname{Hom}_{\mathcal{D}^{b}(Q)}\left(X, \tau^{k} Y[2 k]\right)=0$. Finally, if $d(Y)=0$ then $d\left(\tau^{-1} Y[-2]\right)=-2$ or -1 , and so it is obvious that there is no map from $X$ to $\tau^{-1} Y[-2]$. The case when $k<-1$ is trivial since $d\left(\tau^{k} Y[2 k]\right)$ is negative. If $k>2$, then $d\left(\tau^{k} Y[2 k]\right) \geq k \geq$ 3 , since $d(Y)=0$ or 1 , and so $\operatorname{Hom}_{\mathcal{D}^{b}(Q)}\left(X, \tau^{k} Y[2 k]\right)=0$. Let now $k=2$. If $d(Y)=1$ then $d\left(\tau^{2} Y[4]\right) \in\{3,4,5\}$ and our claim holds. Suppose then that $d(Y)=0$. Then $d\left(\tau^{2} Y[4]\right) \in\{2,3,4\}$. The only nontrivial case is when $d\left(\tau^{2} Y[4]\right)=2$. But this holds if and only if $Y$ is projective and $\overline{\tau Y}$ is an injective-projective module, i.e., $\overline{\tau Y}=P_{a}$ where $a$ must be a source of $Q$. Therefore $\tau^{2} Y[4]=I_{a}[2]$, and $\operatorname{Hom}_{\mathcal{D}^{b}(Q)}\left(X, I_{a}[2]\right) \neq 0$ implies that $d(X)=2$, which is a contradiction since $X \in \mathcal{E}(Q)$.

(2) By Remark 2.1, we have $\operatorname{Hom}_{\mathcal{D}^{b}(Q)}(X, \tau Y[2]) \simeq \operatorname{Hom}_{\mathcal{D}^{b}(Q)}(Y[1], X)$. Suppose $\operatorname{Hom}_{\mathcal{D}^{b}(Q)}(X, Y) \neq 0$. Then, in particular $X \preceq Y$, and so, by transitivity we have $X \preceq Y[1]$. Because $X \neq Y[1]$, we have by antisymmetry that $Y[1] \npreceq X$, which implies that $\operatorname{Hom}_{\mathcal{D}^{b}(Q)}(Y[1], X)=0$.

Proposition 2.3 Let $M$ be an indecomposable object in $\mathcal{C}(Q)$. The full subcategory ${ }^{\perp} M_{Q}^{\perp}$ of $\mathcal{C}(Q)$ whose set of objects is

$$
{ }^{\perp} M_{Q}^{\perp}=\left\{X \in \mathcal{C}(Q) \mid \operatorname{Hom}_{\mathcal{C}(Q)}(X, M)=0=\operatorname{Hom}_{\mathcal{C}(Q)}(M, X)\right\}
$$

is equivalent to $\mathcal{C}\left(Q^{\prime}\right)$ where $Q^{\prime}$ is a disjoint union of quivers of Dynkin type, whose number of vertices is $n-1$.

Firstly, we will show this proposition in the case when $M$ is an indecomposable simple projective $K Q$-module.

Lemma 2.4 Let $M=P_{a}\left(a \in Q_{0}\right)$ be an indecomposable simple projective $K Q$ module. Then ${ }^{\perp} M_{Q}^{\perp}$ is equivalent to $\mathcal{C}\left(Q^{\prime}\right)$ where $Q^{\prime}$ is the full subquiver of $Q$ whose set of vertices is $Q_{0} \backslash\{a\}$.

Proof Let $M=P_{a}$ be an indecomposable simple projective $K Q$-module. Recall that an indecomposable object $X$ in $\mathcal{D}^{b}(Q)$ is of the form $\bar{X}[i]$, where $i \in \mathbb{Z}$ and $\bar{X}$ is an indecomposable module. It is easy to check that the indecomposable objects of ${ }^{\perp} M^{\perp}$ are of the form:

$$
\text { ind } \begin{aligned}
{ }^{\perp} M^{\perp}= & \left\{X \in \text { ind } K Q-\bmod \mid(\underline{\operatorname{dim}} X)_{a}=0\right\} \\
& \cup\left\{\bar{X}[1] \mid \bar{X} \in \text { ind } K Q-\bmod , \bar{X} \text { noninjective, }(\underline{\operatorname{dim} X})_{a}=0\right\} .
\end{aligned}
$$

Let $Q^{\prime}$ be the full subquiver of $Q$ whose set of vertices is $Q_{0} \backslash\{a\}$. Let $\mathcal{S}_{a}$ denote the full subcategory of $K Q$-mod whose set of objects are the $K Q$-modules with no 
support at $a$, and let $\mathcal{D}_{\mathcal{S}_{a}}^{b}$ be the full subcategory of $\mathcal{D}^{b}(Q)$ whose objects are

$$
\operatorname{obj} \mathcal{D}_{\mathcal{S}_{a}}^{b}=\left\{X \in \mathcal{D}^{b}(Q) \mid H^{n}(X) \in \mathcal{S}_{a} \forall n\right\} .
$$

Given that $X \simeq \bigoplus_{n \in \mathbb{Z}} H^{n}(X)[n]$ in the hereditary case (see e.g. [7, Lemma 3.3]), $\mathcal{D}_{\mathcal{S}_{a}}^{b}$ is equivalent to the full subcategory whose collection of objects is $\left\{X \in \mathcal{D}^{b}(Q) \mid\right.$ $\left.X^{n^{a}} \in \mathcal{S}_{a} \forall n\right\}$.

It is easy to check that $\mathcal{D}_{\mathcal{S}_{a}}^{b}$ is triangle equivalent to $\mathcal{D}^{b}\left(Q^{\prime}\right)$. We denote by $G$ this triangle equivalence and we will use it to define a $K$-linear functor $F_{M}$ from ${ }^{\perp} M^{\perp}$ to $\mathcal{C}\left(Q^{\prime}\right)$.

In order to define $F_{M}$ on the objects, note that obj ${ }^{\perp} M^{\perp} \subseteq$ obj $\mathcal{D}_{\mathcal{S}_{a}}^{b}$, by (2). Hence, given $X \in{ }^{\perp} M^{\perp}$, we can define $F_{M}(X)$ to be $G(X)$ regarded as an element of $\mathcal{C}\left(Q^{\prime}\right)$. Note also that if $X \in K Q-\bmod \cap^{\perp} M^{\perp}=\mathcal{S}_{a}$, then $F_{M}(X) \in K Q^{\prime}$-mod. Moreover, it follows from [1, III.2.6 (b)] that $X$ is an injective $K Q$-module if and only if $G(X)$ is an injective $K Q^{\prime}$-module, because $a$ is a sink. Hence $F_{M}$ maps the indecomposable objects of ${ }^{\perp} M^{\perp}$ into the fundamental domain $\mathcal{E}\left(Q^{\prime}\right)$ (recall that we regard the objects of $\mathcal{C}(Q)$ as objects in the fundamental domain $\mathcal{E}(Q))$.

It is enough to define $F_{M}$ on the morphisms between indecomposable objects. So let $X, Y \in$ ind ${ }^{\perp} M^{\perp}$ and $f \in \operatorname{Hom}_{\mathcal{C}(Q)}(X, Y)$. By Proposition 2.2, we have $f \in \operatorname{Hom}_{\mathcal{D}^{b}(Q)}(X, Y)$ or $f \in \operatorname{Hom}_{\mathcal{D}^{b}(Q)}(X, \tau Y[2])$. If $f \in \operatorname{Hom}_{\mathcal{D}^{b}(Q)}(X, Y)$, set $F_{M}(f):=G(f)$. If $f \in \operatorname{Hom}_{\mathcal{D}^{b}(Q)}(X, \tau Y[2])$, we must have $X=\bar{X}$ [1] and $Y=\bar{Y}$. Let $\psi_{Y}$ denote the isomorphism

$$
\operatorname{Hom}_{\mathcal{D}^{b}(Q)}(\bar{X}[1], \tau \bar{Y}[2]) \stackrel{\psi_{Y}}{\longrightarrow} \operatorname{Hom}_{K Q}(\bar{Y}, \bar{X})
$$

and by $\psi_{Y}^{\prime}$ the corresponding isomorphism

$$
\operatorname{Hom}_{\mathcal{D}^{b}\left(Q^{\prime}\right)}\left(\Sigma G(\bar{X}), \Sigma^{2} \tau^{\prime} G(\bar{Y})\right) \stackrel{\psi_{Y}^{\prime}}{\longrightarrow} \operatorname{Hom}_{K Q^{\prime}}(G(\bar{Y}), G(\bar{X}))
$$

in $\mathcal{D}^{b}\left(Q^{\prime}\right)$, where $\Sigma$ denotes the shift functor and $\tau^{\prime}$ the AR-translate in $\mathcal{D}^{b}\left(Q^{\prime}\right)$. We then define $F_{M}(f)$ to be $\psi_{Y}^{\prime-1}\left(G\left(\psi_{Y}(f)\right)\right.$, regarded as an element of $\operatorname{Hom}_{\mathcal{C}\left(Q^{\prime}\right)}\left(F_{M}(X), F_{M}(Y)\right)$.

One can easily see that $F_{M}$ is indeed a functor. Since $G$ is dense, so is $F_{M}$, and it is easy to check that $F_{M}$ is also fully faithful using the definition of $F_{M}$ on the morphisms and the fact that $G$ is an equivalence.

Remark 2.5 Note that the equivalence $F_{M}$ given in the proof above satisfies the following properties, which are going to be useful later:

(1) $d\left(F_{M}(X)\right)=d(X)$ for all $X \in{ }^{\perp} M_{Q}^{\perp}$,

(2) For each $b \in Q_{0} \backslash\{a\}$, the simple $K Q^{\prime}$-module $S_{b}^{\prime}$ is the image of the simple $K Q$-module $S_{b}$,

(3) For each $K Q$-module $X$ in ${ }^{\perp} M_{Q}^{\perp}, \operatorname{supp}\left(F_{M}(X)\right)=\operatorname{supp}(X)$. 
In order to prove Proposition 2.3 we recall the definition of section.

Definition 2.6 [1, VIII.1.2] Let $(\Gamma, \tau)$ be a connected translation quiver. A section of $\Gamma$ is a connected full subquiver $\Sigma$ of $\Gamma$ satisfying the following properties:

i. $\Sigma$ is acyclic.

ii. $\Sigma$ meets each $\tau$-orbit exactly once.

iii. If $x_{0} \rightarrow x_{1} \rightarrow \cdots \rightarrow x_{t}$ is a path in $\Gamma$ with $x_{0}, x_{t} \in \Sigma_{0}$ then $x_{i} \in \Sigma_{0}$, for all $0 \leq i \leq t$.

We can associate a section to an arbitrary indecomposable object $M$ of $\mathcal{D}^{b}(Q)$. Indeed, let $x_{0}$ be the vertex of the AR-quiver $\Gamma$ of $\mathcal{D}^{b}(Q)$ associated to $M$. Recall that $\Gamma=\mathbb{Z} Q$, since $Q$ is of Dynkin type, (cf. [16]). Let $\Sigma=\bigcup_{k} \Sigma^{k}$ be the full subquiver of $\Gamma$ whose set of vertices is defined by

(1) $\Sigma^{0}:=\left\{x_{0}\right\}$,

(2) $\Sigma^{k}:=\left\{y \in \Gamma \mid x \rightarrow y \in \Gamma\right.$ with $x \in \Sigma^{k-1}$ and $\tau y \notin \Sigma^{j}$ for $\left.j<k\right\}$.

Note that $M=\tau^{m} P_{i}$, for some vertex $i$ in $Q$ and some integer $m$, and so $\Sigma=$ $\bigcup_{k=1}^{r} \Sigma^{k}$, where $r$ is the length of the longest unoriented path in $Q$ starting at the vertex $i$.

It is easy to prove that $\Sigma$ is in fact a section and we will call it the section associated to $M$.

The proof of Proposition 2.3 follows easily from Lemma 2.4.

Proof of Proposition 2.3 Let $\Sigma$ be the section associated to $M$ and let $\Omega$ be the quiver obtained from $\Sigma$ by reversing all the arrows. By [1, VIII.1.6], we have $\mathbb{Z} Q \simeq \mathbb{Z} \Omega$, and so $\mathcal{D}^{b}(Q) \simeq \mathcal{D}^{b}(\Omega)$. Let $G$ denote this equivalence. We can assume that the image of $M$ under $G$ is the projective $K \Omega$-module associated to $x_{0}$. This projective module is simple since $x_{0}$ is a sink in $\Omega$. We can easily see that $\mathcal{C}(Q) \simeq \mathcal{C}(\Omega)$ and ${ }^{\perp} M_{Q}^{\perp} \simeq{ }^{\perp} G(M) \stackrel{\perp}{\Omega}$. Because $G(M)$ is a simple projective $K \Omega$-module, it follows from Lemma 2.4 that ${ }^{\perp} G(M) \stackrel{\perp}{\Omega} \simeq \mathcal{C}\left(Q^{\prime}\right)$, where $Q^{\prime}$ is a full subquiver of $\Omega$ with $n-1$ vertices, which finishes the proof.

The main objects of our study are defined as follows.

Definition 2.7 Let $\mathcal{C}$ be an additive category.

(1) A Hom-free set of indecomposable objects of $\mathcal{C}$ is a set $\mathcal{T}$ of indecomposable pairwise non-isomorphic objects of $\mathcal{C}$ such that $\operatorname{Hom}_{\mathcal{C}}(X, Y)=0$ for all $X, Y \in$ $\mathcal{T}, X \neq Y$.

(2) A maximal Hom-free set in $\mathcal{C}$ will be called a Hom-configuration.

We will study Hom-configurations in the quotient category $\mathcal{C}(Q)$.

Example 2.8 Given an arbitrary Dynkin quiver $Q$, the set of simple $K Q$-modules is a Hom-configuration in $\mathcal{C}(Q)$. 
Lemma 2.9 A Hom-free set in $\mathcal{C}(Q)$ is a Hom-configuration if and only if it has $n$ elements.

Proof This follows easily from Proposition 2.3 using induction on the number of vertices of $Q$.

\section{Hom-configurations vs. Hom $\leq 0$-configurations}

In this section we will see that the main object of our study, Hom-configurations in the quotient category $\mathcal{C}(Q)$, are very closely related to Hom $\leq 0$-configurations, a object introduced by Buan-Reiten-Thomas (cf. [10]).

Exceptional sequences are crucial for our study, and are defined as follows.

Definition 3.1 (1) An object $X$ of an Abelian or triangulated category $\mathcal{C}$ is said to be rigid if $\operatorname{Ext}_{\mathcal{C}}^{1}(X, X)=0$. If in addition, the object $X$ is indecomposable then it is said to be exceptional.

(2) An exceptional sequence in $K Q$ - mod is a sequence $\mathcal{E}=\left(E_{1}, \ldots, E_{n}\right)$ of exceptional $K Q$-modules satisfying the following property

$$
\operatorname{Hom}_{K Q}\left(E_{i}, E_{j}\right)=0=\operatorname{Ext}_{K Q}^{1}\left(E_{i}, E_{j}\right), \quad \text { for } j>i .
$$

(3) An exceptional sequence in $\mathcal{D}^{b}(Q)$ is a sequence of exceptional objects satisfying the following property

$$
\operatorname{Ext}_{\mathcal{D}^{b}(Q)}^{m}\left(E_{i}, E_{j}\right)=0, \quad \text { for } j>i \text { and } m \in \mathbb{Z} .
$$

In order to simplify the exposition, we use the reverse of the usual convention for the order of an exceptional sequence.

Lemma 3.2 An exceptional sequence in $\mathcal{D}^{b}(Q)$ can also be defined to be a sequence $\left(X_{1}, \ldots, X_{n}\right)$ of indecomposable objects such that $\left(\bar{X}_{1}, \ldots, \bar{X}_{n}\right)$ is an exceptional sequence in $K Q$ - mod.

Proof Suppose $\left(X_{1}, \ldots, X_{n}\right)$ is an exceptional sequence in $\mathcal{D}^{b}(Q)$ as defined in Definition 3.1(3). Since for each $i \in[n], X_{i}$ is indecomposable, we have $X_{i}=\bar{X}_{i}\left[t_{i}\right]$, for some integer $t_{i}$. In other words, $\bar{X}_{i}=X_{i}\left[-t_{i}\right]$, for all $i$. Note also that we have $\operatorname{Ext}_{K Q}^{k}\left(\bar{X}_{i}, \bar{X}_{j}\right) \simeq \operatorname{Ext}_{\mathcal{D}^{b}(Q)}^{k}\left(\bar{X}_{i}, \bar{X}_{j}\right)$, for $k=0,1$ and $j>i$, since $\bar{X}_{j}, \bar{X}_{i}$ are $K Q$ modules. Hence, for $j>i$ and $k=0,1$, we have

$$
\begin{aligned}
\operatorname{Ext}_{K Q}^{k}\left(\bar{X}_{i}, \bar{X}_{j}\right) & \simeq \operatorname{Hom}_{\mathcal{D}^{b}(Q)}\left(X_{i}\left[-t_{i}\right], X_{j}\left[k-t_{j}\right]\right) \\
& \simeq \operatorname{Hom}_{\mathcal{D}^{b}(Q)}\left(X_{i}, X_{j}\left[k-t_{j}+t_{i}\right]\right)=0,
\end{aligned}
$$

by Definition 3.1 .

Conversely, suppose $\left(X_{1}, \ldots, X_{n}\right)$ is a sequence of indecomposable objects in $\mathcal{D}^{b}(Q)$ such that $\left(\bar{X}_{1}, \ldots, \bar{X}_{n}\right)$ is an exceptional sequence in $K Q$-mod. Then we 
have $\operatorname{Hom}_{\mathcal{D}^{b}(Q)}\left(\bar{X}_{i}, \bar{X}_{j}[k]\right)=0$, for every integer $k$ and $j>i$, by assumption in the case when $k=0,1$ and because $\bar{X}_{j}$ and $\bar{X}_{i}$ are $K Q$-modules, in the case when $k \in \mathbb{Z} \backslash\{0,1\}$. Using the same argument as used above, we easily deduce that $\left(X_{1}, \ldots, X_{n}\right)$ is an exceptional sequence in $\mathcal{D}^{b}(Q)$ as defined in Definition 3.1.

In [10], Buan, Reiten and Thomas define a new object in the bounded derived category $\mathcal{D}^{b}(Q)$ of an arbitrary hereditary Artin algebra, called a Hom $\leq 0$-configuration.

Definition 3.3 [10] An object $X \in \mathcal{D}^{b}(Q)$ is a Hom $\leq 0$-configuration if it satisfies the following axioms:

(1) $X$ has $n$ indecomposable pairwise non-isomorphic summands $X_{1}, \ldots, X_{n}$, and they are rigid.

(2) $\operatorname{Hom}_{\mathcal{D}^{b}(Q)}\left(X_{i}, X_{j}\right)=0$ for $i \neq j$.

(3) $\operatorname{Ext}_{\mathcal{D}^{b}(Q)}^{k}(X, X)=0$ for $k<0$.

(4) The indecomposable direct summands can be ordered into an exceptional sequence.

Our aim is to prove that $\mathrm{Hom}_{\leq 0}$-configurations contained in $\mathcal{E}(Q)$ are precisely the Hom-configurations in $\mathcal{C}(Q)$.

Lemma 3.4 Let $\mathcal{T}$ be a Hom-configuration in $\mathcal{C}(Q)$, and let $X, Y \in \mathcal{T}$. We have $\operatorname{Ext}_{\mathcal{D}^{b}(Q)}^{i}(X, Y)=0$, for $i \leq 0$.

Proof Note that $X, Y \in \mathcal{E}$. So, we have

$$
0=\operatorname{Hom}_{\mathcal{C}(Q)}(X, Y) \simeq \operatorname{Hom}_{\mathcal{D}^{b}(Q)}(X, Y) \oplus \operatorname{Hom}_{\mathcal{D}^{b}(Q)}(X, \tau Y[2]) .
$$

Hence $\operatorname{Hom}_{\mathcal{D}^{b}(Q)}(X, Y)=0$. We also have $\operatorname{Hom}_{\mathcal{C}(Q)}(Y, X)=0$, which implies that $\operatorname{Hom}_{\mathcal{D}^{b}(Q)}(Y, \tau X[2])=0$, and so $\operatorname{Ext}_{\mathcal{D}^{b}(Q)}^{-1}(X, Y)=0$, by Remark 2.1. For $i \leq-2$, we see that $Y[i]$ has negative degree, as $Y$ lies in $\mathcal{E}$. Therefore $\operatorname{Ext}_{\mathcal{D}^{b}(Q)}^{i}(X, Y)=0$, for $i \leq-2$, since $X$ has degree 0 or 1 .

Remark 3.5 (1) Let $P$ be an indecomposable projective $K Q$-module. If $\operatorname{Hom}_{\mathcal{D}^{b}(Q)}(P, X) \neq 0$ then $X \in K Q$-mod.

(2) Any non-zero $K Q$-module has a non-zero morphism to an indecomposable injective module.

(3) Let $P$ be an indecomposable projective $K Q$-module. We have $P \preceq I$ for any indecomposable injective module $I$.

Proof We just prove (3). Let $P_{i}$ be the indecomposable projective $K Q$-module associated to vertex $i$ in $Q$, and let $Q^{\prime}$ be the quiver obtained from $Q$ by orienting all the arrows away from $i$. Note that the indecomposable projective $K Q^{\prime}$-module $P_{i}^{\prime}$ associated to the vertex $i$ in $Q^{\prime}$ is sincere. We have a derived equivalence between the two module categories, i.e., $\mathcal{D}^{b}(Q) \simeq \mathcal{D}^{b}\left(Q^{\prime}\right)$, and we can assume that this equivalence identifies the projectives at $i$ for the two quivers, i.e., $G\left(P_{i}\right)=P_{i}^{\prime}$. 
Since $P_{i}^{\prime}$ is sincere, we have $\operatorname{Hom}_{K Q^{\prime}}\left(P_{i}^{\prime}, I^{\prime}\right) \neq 0$, for all indecomposable injective $K Q^{\prime}$-modules $I^{\prime}$. Hence, in particular, $P_{i}^{\prime} \preceq I^{\prime}$.

Note that under the equivalence $G$ we find that, for an arbitrary indecomposable injective $K Q$-module $I, G(I)$ is either injective or it lies in $K Q^{\prime}-\bmod [1]$. Therefore, as we have seen above, $G\left(P_{i}\right)=P_{i}^{\prime} \preceq G(I)$, which implies that $P_{i} \preceq I$, for any indecomposable injective $K Q$-module.

Proposition 3.6 Let $\mathcal{T}$ be a Hom-configuration in $\mathcal{C}(Q)$ (regarded as lying in $\mathcal{E}(Q)$ ). If we order the elements of $\mathcal{T}$ respecting the total order $\leq$ (i.e., order the elements from left to right in the AR-quiver), we obtain an exceptional sequence in $\mathcal{D}^{b}(Q)$.

Proof Let $\mathcal{T}=\left\{X_{1}, \ldots, X_{n}\right\}$ be a Hom-configuration of $\mathcal{C}(Q)$ ordered with respect to the total order $\leq$. We want to check that

$$
\operatorname{Ext}_{\mathcal{D}^{b}(Q)}^{m}\left(X_{i}, X_{j}\right)=0
$$

for $j>i$ and for any integer $m$.

Note that (3) holds for $m \leq 0$, by Lemma 3.4. (3) also holds for $m>2$ since $X_{i}$ has degree 0 or 1 and $X_{j}[m]$ has degree $\geq 3$.

Let us check the case when $m=1$. Given $i, j \in[n]$ with $i<j$, we have $\operatorname{Ext}_{\mathcal{D}^{b}(Q)}^{1}\left(X_{i}, X_{j}\right) \simeq \operatorname{Hom}_{\mathcal{D}^{b}(Q)}\left(X_{j}, \tau X_{i}\right)$ by Serre duality. If this is non-zero, then we have in particular that there is a path from $X_{j}$ to $\tau X_{i}$ in the AR-quiver of $\mathcal{D}^{b}(Q)$, which implies that $X_{j} \leq \tau X_{i}$. On the other hand, $\tau X_{i} \leq X_{i}$, and so by transitivity, we have $X_{j} \leq X_{i}$. Since $j>i$, we have $X_{i} \leq X_{j}$, so $X_{i}=X_{j}$ by antisymmetry, a contradiction. Hence, (3) holds for $m=1$.

Finally, let us check for $m=2$. Note that $X_{i} \leq X_{j}$, and so $X_{j} \npreceq X_{i}$, i.e., there is no path from $X_{j}$ to $X_{i}$ in the AR-quiver of $\mathcal{D}^{b}(Q)$.

We have $X_{i}=\tau^{-l}\left(P_{a}\right)$, for some natural number $l$ and some indecomposable projective $K Q$-module $P_{a}$ ( $a$ denotes the vertex corresponding to the projective module).

Suppose $\operatorname{Ext}_{\mathcal{D}^{b}(Q)}^{2}\left(X_{i}, X_{j}\right)=\operatorname{Hom}_{\mathcal{D}^{b}(Q)}\left(P_{a}, \tau^{l} X_{j}[2]\right) \neq 0$. By Remark 3.5(1), $\tau^{l} X_{j}[2]$ is a $K Q$-module, i.e., $\tau^{l} X_{j}$ has degree -2 . It follows from Remark 3.5(2) that there is a path from $\tau^{l} X_{j}$ to $I[-2]$, for some indecomposable injective module $I$, i.e., $\tau^{l} X_{j} \preceq I[-2]$. We also have $I[-2] \preceq P[-1]$, where $P$ is the indecomposable projective such that $\operatorname{soc} I \simeq P / \operatorname{rad} P$, in other words, $\tau P=I[-1]$. By Remark 3.5(3), we have $P[-1] \preceq I_{a}[-1]$. We also have $I_{a}[-1] \preceq P_{a}$. By transitivity we can conclude that $\tau^{l} X_{j} \preceq P_{a}$, and so $X_{j}=\tau^{-l}\left(\tau^{l} X_{j}\right) \preceq \tau^{-l} P_{a}=X_{i}$, which is a contradiction.

Theorem 3.7 Hom-configurations in $\mathcal{C}(Q)$ are in $1-1$ correspondence with Hom $_{\leq 0}$-configurations contained in $\mathcal{E}(Q)$.

Proof Let $\mathcal{T}$ be a Hom-configuration of $\mathcal{C}(Q)$. By Lemma 2.9 $\mathcal{T}$ has $n$ elements, and every element of $\mathcal{T}$ is rigid, since $Q$ is of Dynkin type. Properties 2 and 3 of Definition 3.3 follow from Lemma 3.4 and the definition of Hom-configuration and property 4 follows from Proposition 3.6. Conversely, suppose $\mathcal{T}$ is a $\mathrm{Hom}_{\leq 0}$-configuration of $\mathcal{D}^{b}(Q)$ contained in $\mathcal{E}(Q)$. Then by property 1 , it has $n$ elements, and for any pair 
of objects $X, Y$ of $\mathcal{T}$ we have

$$
\operatorname{Hom}_{\mathcal{C}(Q)}(X, Y)=\operatorname{Hom}_{\mathcal{D}^{b}(Q)}(X, Y) \oplus \operatorname{Hom}_{\mathcal{D}^{b}(Q)}(X, \tau Y[2]),
$$

since $X, Y$ lie in $\mathcal{E}$. Both summands are zero, by properties 2,3 and by Remark 2.1. Hence $\mathcal{T}$ is a Hom-free set with $n$ elements, and so the result follows from Lemma 2.9 .

Note that the full subcategory $\mathcal{D}_{\leq 1}^{(\geq 0)-}$ of $\mathcal{D}^{b}(Q)$ whose indecomposables are in $K Q-\bmod [i] \backslash\left\{P_{i} \mid i \in[n]\right\}$ with $i=0,1$, which was considered in [10], is just a different fundamental domain for the action of $\tau[2]$ in $\mathcal{D}^{b}(Q)$.

Remark 3.8 Let $\mathcal{T}=\left\{X_{1}, \ldots, X_{r}, X_{r+1}[1], \ldots, X_{n}[1]\right\}$ be a Hom-configuration in $\mathcal{C}(Q)$ lying in $\mathcal{E}(Q)$, where $X_{i} \in K Q$ - $\bmod$ for $i=1, \ldots, n$ and $X_{j} \in K Q$ - $\bmod \backslash \mathcal{I}$, for $j \geq r+1$.

It is easy to check that the map $\mu$ sending $\mathcal{T}$ to $\left\{\tau^{-1} X_{r+1}, \ldots, \tau^{-1} X_{n}, X_{1}[1], \ldots\right.$, $\left.X_{r}[1]\right\}$ gives a 1-1 correspondence between Hom-configurations in $\mathcal{C}(Q)$ (regarded as lying in $\mathcal{E}(Q))$ and $\mathrm{Hom}_{\leq 0}$-configurations contained in $\mathcal{D}_{\leq 1}^{(\geq 0)-}$.

We will refer to this map $\mu$ later in Sect. 5 .

\section{Sincere Hom-free sets in $K Q$-mod}

This section is devoted to the study of the set of modules of a Hom-configuration. We prove that the restriction of the Hom-configurations in $\mathcal{C}(Q)$ to $K Q$ - mod is precisely the set of sincere Hom-free sets.

Some of the proofs will rely on using reflection functors, which correspond to changing the orientation in the quiver $Q$, to reduce to the case when we have a simple projective module, so we can use Lemma 2.4.

Given a sink or a source $i$ of the quiver $Q$, we denote by $\sigma_{i}(Q)$ the quiver obtained from $Q$ by reversing all the arrows incident to $i$. We denote by $R_{i}$ the (simple) reflection functor associated to a sink $i$ and by $R_{j}^{-}$the simple reflection functor associated to a source $j$. If $i$ is a sink of $Q$, the functor $R_{i}$ gives an equivalence between $\mathcal{D}^{b}(Q)$ and $\mathcal{D}^{b}\left(\sigma_{i}(Q)\right)$, and the inverse is given by $R_{i}^{-}$. Because $R_{i}$ and $R_{i}^{-}$commute with $\tau$ and [-], these functors induce equivalences between $\mathcal{C}(Q)$ and $\mathcal{C}\left(\sigma_{i}(Q)\right)$. If we have a sequence $i_{1}, \ldots, i_{k}$ of vertices of $Q$ such that each $i_{j}$ is a sink in $\sigma_{i_{j-1}} \ldots \sigma_{i_{1}}(Q)$, and $R$ is the sequence of reflections $R_{i_{k}} \ldots R_{i_{1}}$, we denote by $\sigma_{R}(Q)$ the quiver $\sigma_{i_{k}} \ldots \sigma_{i_{1}}(Q)$, for simplicity.

We have the following useful description of the image of an indecomposable object of $\mathcal{D}^{b}(Q)$ under these reflection functors:

Let $i$ be a sink (source) of $Q$, and $M[j]$ an indecomposable object of $\mathcal{D}^{b}(Q)$. If $M \neq S_{i}$, then $R_{i}(M[j])=N[j]\left(R_{i}^{-}(M[j])=N[j]\right)$, where $N$ is the indecomposable $K \sigma_{i}(Q)$-module whose dimension vector is $\underline{\operatorname{dim}}(N)=s_{i}(\underline{\operatorname{dim}} M)$, where $s_{i}$ is the simple reflection associated to the simple root $\alpha_{i}$. If $M=S_{i}$, then $R_{i}(M[j])=$ $M[j-1]\left(R_{i}^{-}(M[j])=M[j+1]\right)$. 
Remark 4.1 (1) Given $X \in \mathcal{C}(Q)$, there exists a composition of reflection functors $R_{i_{k}} \ldots R_{i_{1}}$, where $i_{j}$ is a sink in $\sigma_{i_{j-1}} \ldots \sigma_{i_{1}}(Q)$ for each $2 \leq j \leq k$ such that $R_{i_{k}} \ldots R_{i_{1}}(X)$ is a simple projective $K\left(\sigma_{i_{k}} \ldots \sigma_{i_{1}}(Q)\right)$-module.

(2) Given a set $\mathcal{T}$ of $n$ objects in $\mathcal{C}(Q)$ and a sink $i$ in $Q, \mathcal{T}$ is a Hom-configuration in $\mathcal{C}(Q)$ if and only if $R_{i}(\mathcal{T})$ is a Hom-configuration in $\mathcal{C}\left(\sigma_{i}(Q)\right)$.

Proof Part (1) is a well known result, but we will give a specific sequence of reflection functors which will be useful later.

Consider the set of objects $\{Y \in \mathcal{E}(Q) \mid Y \preceq X\}$. Let $\left\{Y_{1}, \ldots, Y_{k}\right\}$ be the ordering of the elements of this set with respect to the total order $\leq$. Given that $Y_{1}$ is $\preceq$-minimal, $Y_{1}$ must be a simple projective $K Q$-module. Let $i_{1}$ be the sink of $Q$ associated to $Y_{1}$, i.e., $Y_{1}=P_{i_{1}}$. Note that $Y_{2}$ is $\preceq$-minimal in $\mathcal{E}\left(\sigma_{i_{1}}(Q)\right)$, i.e., $Y_{2}$ is a simple projective $K \sigma_{i_{1}}(Q)$-module. Let $i_{2}$ be the corresponding sink in $\sigma_{i_{1}}(Q)$. Proceeding this way, we get the composition $R_{i_{k}} \ldots R_{i_{2}} R_{i_{1}}$ of reflection functors. Clearly this composition maps $X$ to a simple projective $K \sigma_{R}(Q)$-module.

Part (2) follows easily from the fact that $R_{i}$ is an equivalence and it commutes with $\tau$ and the shift functor.

Lemma 4.2 (1) [24, Theorem 3] The set of the simple KQ-modules is the unique Hom-configuration of $\mathcal{C}(Q)$ consisting of modules.

(2) Any Hom-configuration of $\mathcal{C}(Q)$ has at least one $K Q$-module.

Proof Part (1) was proved by Ringel (cf. [24, Theorem 3]) but we will give an alternative proof, which will be by induction on $n$, the number of vertices of $Q$. The case when $n=1$ is very easy to check. Let $\mathcal{T}$ be a Hom-configuration consisting of modules in $\mathcal{C}(Q)$, where $\left|Q_{0}\right|=n$. First suppose that $\mathcal{T}$ contains a simple projective module $S_{i}$. We will use the equivalence $F_{S_{i}}$ between ${ }^{\perp} S_{i}^{\perp}$ and $\mathcal{C}\left(Q^{\prime}\right)$, where $Q^{\prime}=Q \backslash\{i\}$ defined in Sect. 2 (for its definition and some of its properties, see proof of Lemma 2.4 and Remark 2.5). Note that $F_{S_{i}}\left(\mathcal{T} \backslash S_{i}\right)$ is a Hom-configuration in $\mathcal{C}\left(Q^{\prime}\right)$ and it consists only of $K Q^{\prime}$-modules, by Remark 2.5(1). It follows by induction that these $K Q^{\prime}$-modules are the simple $K Q^{\prime}$-modules, and so we have $\mathcal{T}=\left\{S_{1}, \ldots, S_{n}\right\}$, by Remark 2.5(2).

Suppose now that $\mathcal{T}$ does not contain any simple projective module. Let $X$ be a minimal element of $\mathcal{T}$ with respect to the partial order $\preceq$. Let $R$ be the composition of reflection functors described in the proof of Remark 4.1(1). Observe that $R$ maps $X$ to a simple projective $K \sigma_{R}(Q)$-module and if $Y \in K Q$-mod with $Y \npreceq X$ then $R(Y) \in K \sigma_{R}(Q)$ - mod. The same holds for $R^{\prime}:=R_{i_{k-1}} \ldots R_{i_{2}} R_{i_{1}}$, i.e., $R^{\prime}(Y) \in K \sigma_{R^{\prime}}(Q)$-mod. So, in particular, $R(\mathcal{T})$ lies in $K \sigma_{R}(Q)$-mod, due to the choice of $X$. Moreover, by Remark 4.1(2), $R(\mathcal{T})$ is a Hom-configuration in $\mathcal{C}\left(\sigma_{R}(Q)\right)$ and it contains a simple projective module. Hence, $R(\mathcal{T})$ is the set of simple modules in $K \sigma_{R}(Q)$ - mod. However, if one considers the simple injective $K \sigma_{R}(Q)$-module $S^{\prime}$ corresponding to $R_{i_{k}}$, we know there is an element $Y$ of $\mathcal{T}$ such that $R(Y)=S^{\prime}$, and $R_{i_{k-1}} \ldots R_{i_{1}}(Y)$ has degree 0 but $R_{i_{k-1}} \ldots R_{i_{1}}(Y)=R_{i_{k}}^{-1}\left(S^{\prime}\right)=S^{\prime}$ [1], a contradiction.

To prove (2), suppose $\mathcal{T}$ is a Hom-configuration of $\mathcal{C}(Q)$ whose objects lie in $\operatorname{ind}((\bmod K Q \backslash \mathcal{I})[1])$. Then $\mathcal{T}$ must be the set $\{S[1] \mid S$ simple module $\}$, since otherwise $\mathcal{T}[-1]=\{M[-1] \mid M \in \mathcal{T}\}$ would be a Hom-configuration consisting of mod- 
ules which is not the set of the simple modules, contradicting (1). However, since $Q$ is a Dynkin quiver, it must have a source $i$. Hence $S_{i}=I_{i}$ is injective, a contradiction.

In order to extend a sincere Hom-free set in $K Q$-mod to a Hom-configuration by adding indecomposable objects of degree 1 , we use the notion of perpendicular category. If $\mathcal{T}$ is a set of indecomposable modules, the perpendicular category is defined by

$$
\mathcal{T}^{\perp}=\left\{M \in K Q-\bmod \mid \operatorname{Hom}_{K Q}(X, M)=0, \operatorname{Ext}_{K Q}^{1}(X, M)=0, \forall X \in \mathcal{T}\right\} .
$$

If $Q$ is a Dynkin quiver and $X$ is an indecomposable $K Q$-module, it is well known that $X_{Q}^{\perp}$ is equivalent to $K Q^{\prime}$ - $\bmod$ where $Q^{\prime}$ is a quiver with no oriented cycles and $\left|Q_{0}\right|-1$ vertices. Note that the functor from $K Q^{\prime}-\bmod$ to $K Q-\bmod$ is exact and induces isomorphisms on both Hom and Ext. We refer the reader to [25, Theorem 2.3] for more details.

Remark 4.3 Let $\mathcal{T}$ be a Hom-free set in $K Q$-mod. Then $\mathcal{T}_{Q}^{\perp} \simeq K Q^{\prime}$ - $\bmod$ for some quiver $Q^{\prime}$ which is a union of Dynkin quivers and it has $\left|Q_{0}\right|-|\mathcal{T}|$ vertices.

Proof This follows immediately from Theorem 2.5 in [25] and Proposition 3.6.

Proposition 4.4 Let $\mathcal{T}$ be a sincere Hom-free set in $K Q$-mod. There exists a unique Hom-configuration of $\mathcal{C}(Q)$ whose restriction to $K Q$ - $\bmod$ is $\mathcal{T}$.

Proof Let $\mathcal{T}=\left\{X_{1}, \ldots, X_{k}\right\}$ be a sincere Hom-free set in $K Q$-mod. For each injective module $I$, there exists $i \in[k]$ such that $\operatorname{Hom}_{K}\left(X_{i}, I\right) \neq 0$, since $\mathcal{T}$ is sincere. Hence the injective $K Q$-modules do not lie in $\mathcal{T}^{\perp}$.

We claim that given $Y \in \operatorname{ind}(K Q-\bmod \backslash \mathcal{I}), Y[1]$ lies in ${ }^{\perp} \mathcal{T}^{\perp}$ if and only if $Y \in \mathcal{T}^{\perp}$. Indeed, given $X \in \mathcal{T}$, we have $\operatorname{Hom}_{\mathcal{C}(Q)}(X, Y[1]) \simeq \operatorname{Hom}_{\mathcal{D}^{b}(Q)}(X, Y[1]) \simeq$ $\operatorname{Ext}_{K Q}^{1}(X, Y)$, and $\operatorname{Hom}_{\mathcal{C}(Q)}(Y[1], X)=\operatorname{Hom}_{\mathcal{D}^{b}(Q)}(Y[1], \tau X[2]) \simeq \operatorname{Hom}_{K Q}(X, Y)$, and so our claim holds.

We know $\mathcal{T}^{\perp} \simeq K Q^{\prime}$ - mod, for some Dynkin quiver $Q^{\prime}$ with $n-k$ vertices.

Consider $U=\left\{S[1] \mid S\right.$ simple object in $\left.\mathcal{T}^{\perp}\right\}$. By the first part of this proof, $U \subseteq$ ${ }^{\perp} \mathcal{T}^{\perp}$ and so $\mathcal{T}^{\prime} \sqcup U$ is a Hom-free set in $\mathcal{C}(Q)$. Since $U$ has $n-k$ elements, $\mathcal{T}^{\prime} \sqcup U$ is indeed a Hom-configuration in $\mathcal{C}(Q)$.

To prove the uniqueness let $V$ be a set of elements in $\operatorname{ind}(K Q$ - $\bmod \backslash \mathcal{I})[1]$ such that $\mathcal{T}^{\prime} \sqcup V$ is a Hom-configuration in $\mathcal{C}(Q)$. Then it follows from the first part of the proof that $V[-1]$ is a Hom-free set in $\mathcal{T}^{\perp}$ and it contains $n-k$ elements. But $\mathcal{T}^{\perp} \simeq K Q^{\prime}$-mod, where $Q^{\prime}$ has $n-k$ vertices, so it follows from 4.2 (1) that $V$ must be the shift of the simple objects in $\mathcal{T}^{\perp}$.

We remark that the proof gives us an explicit way to extend a sincere Hom-free set to a Hom-configuration, by simply taking the perpendicular category of the sincere Hom-free set and picking the shift of the simple objects in this category. 
Example 4.5 Consider the quiver $Q: 4 \longrightarrow 3 \longleftrightarrow 2 \longrightarrow 1$ of type $A_{4}$.

We denote an indecomposable module with dimension vector given by $\left(i_{1}, i_{2}, i_{3}, i_{4}\right)$ by listing the vertices of the simple modules in its support.

Let $\mathcal{T}=\{34,12\}$. Then obj $\mathcal{T}^{\perp}=\{1,123,23\}$ and the simple objects of this subcategory are 1 and 23 . So $\mathcal{U}=\{1[1], 23[1]\}$ and the unique Hom-configuration whose set of modules is $\mathcal{T}$ is $\mathcal{T} \sqcup \mathcal{U}=\{12,34,1[1], 23[1]\}$. Note that $\mathcal{T} \sqcup \mathcal{U}$ is not the unique Hom-configuration containing $\mathcal{T}$. For instance, $\{12,34,23,123[1]\}$ is also a Hom-configuration, but it contains a module not in $\mathcal{T}$.

Lemma 4.6 Let $\mathcal{T}$ be a Hom-configuration in $\mathcal{C}(Q)$ such that $\mathcal{T}^{\prime}:=\left.\mathcal{T}\right|_{K Q \text { - } \bmod }$ is sincere, and let $i$ be a source in $Q$. Then $\left.R_{i}^{-}(\mathcal{T})\right|_{K \sigma_{i}(Q)-\bmod }$ is also sincere.

Proof We will use the following notation for simplicity: $\mathcal{U}=R_{i}^{-}(\mathcal{T}), \mathcal{U}^{\prime}=R_{i}^{-}(\mathcal{T}) \cap$ $K \sigma_{i}(Q)-\bmod$ and $\mathcal{U}^{\prime \prime}=\mathcal{U} \backslash \mathcal{U}^{\prime}$

Note that all $K Q$-modules except $S_{i}$ are mapped to $K \sigma_{i}(Q)$-modules via $R_{i}^{-}$, and moreover, the support on all the vertices other than $i$ remains unchanged, so we only need to analyze what happens to the support on vertex $i$.

Suppose $S_{i}=I_{i}$ belongs to $\mathcal{T}$. Let $j$ be a neighbor of $i$ in $Q$. There is a $K Q$ module $Y$ in $\mathcal{T}^{\prime}$ with $(\operatorname{dim} Y)_{j} \neq 0$, by hypothesis. On the other hand, we must have $(\underline{\operatorname{dim}} Y)_{i}=0$, otherwise $\operatorname{Hom}\left(Y, I_{i}\right) \neq 0$, which contradicts the fact that $\mathcal{T}$ is Homfree. Therefore $\left(\underline{\operatorname{dim}} R_{i}^{-}(Y)\right)_{i} \neq 0$ and the sincerity is preserved, as we wanted.

Suppose now that $I_{i}$ does not lie in $\mathcal{T}$. Assume, for a contradiction, that $\mathcal{U}^{\prime}$ is not sincere, i.e., no $K \sigma_{i}(Q)$-module in $R_{i}^{-}(\mathcal{T})$ has support on $i$. Let $I^{i}$ be the indecomposable injective $K \sigma_{i}(Q)$-module associated to the vertex $i$. Then we have $\operatorname{Hom}_{K \sigma_{i}(Q)}\left(X, I^{i}\right)=0$, for all $X \in \mathcal{U}^{\prime}$. We also have $\operatorname{Ext}_{K \sigma_{i}(Q)}^{1}\left(X, I^{i}\right)=$ $\operatorname{Hom}\left(X, I^{i}[1]\right)=0$, since $I^{i}$ is injective and $d(X)=0$. Hence $I^{i} \in \mathcal{U}^{\prime \perp}$. By Remark 4.3, we have $\mathcal{U}^{\prime^{\perp}} \simeq{ }^{G} K Q^{\prime}$ - $\bmod$, where $Q^{\prime}$ is a union of Dynkin quivers and it has $n-\left|\mathcal{U}^{\prime}\right|$ vertices. Let $G\left(I^{i}\right) \rightarrow S \rightarrow 0$ be a surjection in $K Q^{\prime}$ - $\bmod$ (note that every module maps to a simple), and $K$ its kernel. Then we have a short exact sequence $0 \rightarrow G^{-1}(K) \rightarrow I^{i} \rightarrow G^{-1}(S) \rightarrow 0$ in $\mathcal{U}^{\perp}$. Since $I^{i}$ is injective, so is $G^{-1}(S)$. On the other hand, the image of $\mathcal{U}^{\prime \prime}[-1]$ under $G$ is the set of simple $K Q^{\prime}$-modules and $\mathcal{U}^{\prime \prime}[-1]$ does not contain any injective $K \sigma_{i}(Q)$-module (see proof of Proposition 4.4), so $G^{-1}(S)$ is not injective, a contradiction.

Proposition 4.7 The set of modules of any Hom-configuration in $\mathcal{C}(Q)$ is sincere.

Proof We prove this by induction on $n$. The proposition is trivial in the case when $n=1$. Now suppose the proposition holds for $n-1$ and let $\mathcal{T}$ be a Hom-configuration in $\mathcal{C}(Q)$ with $\left|Q_{0}\right|=n$. If $\mathcal{T}$ has a simple projective $K Q$-module $P_{i}$, then the set modules of the Hom-configuration $F_{P_{i}}\left(\mathcal{T} \backslash P_{i}\right)$ in $\mathcal{C}\left(Q^{\prime}\right)$, with $Q^{\prime}=Q \backslash\{i\}$, is sincere by induction. So the set of modules in $\mathcal{T} \backslash P_{i}$ has support on every vertex of $Q$ except $i$, by Remark 2.5(3). But $P_{i}$ has support on $i$, so $\left.\mathcal{T}\right|_{K Q \text { - mod }}$ is sincere. Suppose now that $\mathcal{T}$ does not have any simple projective module. Let $X$ be an element of $\mathcal{T}$. We know that there is a sequence of reflections $R_{i_{1}}, \ldots, R_{i_{k}}$ such that $R_{i_{k}} \ldots R_{i_{1}}(X)$ is a simple projective $K \sigma(Q)$-module, with $\sigma(Q)=\sigma_{i_{k}} \ldots \sigma_{i_{1}}(Q)$. It follows from what 
was proved above that $\left.R_{i_{k}} \ldots R_{i_{1}}(\mathcal{T})\right|_{K \sigma(Q) \text {-mod }}$ is sincere, and so the proposition follows immediately from Lemma 4.6.

Theorem 4.8 Let $\beta$ be the map from the collection of sincere Hom-free sets in $K Q-\bmod$ to the collection of Hom-configurations in $\mathcal{C}(Q)$ defined as follows. Given a sincere Hom-free set $\mathcal{T}$ in $K Q$ - $\bmod , \beta(\mathcal{T}):=\mathcal{T} \sqcup U$, where

$$
\mathcal{U}=\left\{S[1] \mid \text { S simple object in } \mathcal{T}^{\perp}\right\} .
$$

Then this map is a bijection, and its inverse is given by the restriction to $K Q$-mod.

Proof This follows immediately from Propositions 4.4 and 4.7.

\section{Positive noncrossing partitions}

Let $W$ be a finite Coxeter group, $S$ the set of simple generators of $W$ and $T$ the set of reflections. Fix a Coxeter element $c$ in $W$. For $w \in W$, let $l_{T}(w)$ denote the absolute length of $w$, which is the minimum length of $w$ written as a product of reflections. Given $w \in W$, we call a minimum length expression for $w$ written as a product of reflections as $T$-reduced expression. The absolute length naturally induces a partial order $\leq_{T}$ on $W$, which will be called the absolute order.

Definition 5.1 The absolute order $\leq_{T}$ is defined by

$$
u \leq_{T} v \quad \Leftrightarrow \quad l_{T}(v)=l_{T}(u)+l_{T}\left(u^{-1} v\right),
$$

for all $u, v \in W$.

Another way to define absolute order is by saying that $u \leq_{T} v$ if there is a $T$-reduced expression for $v$ in which an expression for $u$ appears as a prefix.

Definition $5.2[2,5]$ A noncrossing partition associated to $W$ is an element $w \in$ $W$ satisfying $1 \leq_{T} w \leq_{T} c$. The poset of noncrossing partitions associated to $W$ is denoted by $N C(W)$.

We will state here a lemma proved by Reading [21] which will be useful later.

Lemma 5.3 [21, Lemma 5.2] Let $x \leq_{T} c$, s be a simple reflection and $W_{S \backslash\{s\}}$ be the standard parabolic subgroup generated by every simple reflection but $s$. Then the following are equivalent:

(1) $x \in W_{S \backslash\{s\}}$.

(2) Every reflection $t$ in any $T$-reduced expression for $x$ lies in $W_{S \backslash\{s\}}$.

Definition 5.4 [21] A noncrossing partition associated to $W$ which is not contained in any proper standard parabolic subgroup is said to be a positive noncrossing partition. The subset of $N C(W)$ consisting of positive noncrossing partitions will be denoted by $\mathrm{NC}^{+}(W)$. 
Let now $W_{Q}$ be the Coxeter group associated to the simply laced Dynkin quiver $Q$, and fix a Coxeter element $c=s_{i_{1}} \ldots s_{i_{n}}$ adapted to the quiver $Q$ with respect to sinks, i.e., $i_{1}$ is a sink of $Q$, and $i_{k}$ is a sink of the quiver $\sigma_{i_{k-1}} \ldots s_{i_{2}} s_{i_{1}}(Q)$, for each $k \geq 2$. The cardinality of $N C\left(W_{Q}\right)$ is given by the Catalan number associated to $Q$ (cf. [2]).

It was proved in [21] that the number of positive noncrossing partitions is given by the so called positive Fuss-Catalan number $C^{+}\left(W_{Q}\right)$, which is defined as (see [14])

$$
\prod_{i=1}^{n} \frac{e_{i}+h-1}{e_{i}+1}
$$

where $h$ is the Coxeter number of $W_{Q}$ and $e_{1}, \ldots, e_{n}$ its exponents.

The following table (cf. Table 4 in [14]) shows the explicit formulas for Dynkin type.

\begin{tabular}{llllll}
\hline$Q$ & $A_{n}$ & $D_{n}$ & $E_{6}$ & $E_{7}$ & $E_{8}$ \\
\hline$C^{+}\left(W_{Q}\right)$ & $\frac{1}{n+1}\left(\begin{array}{c}2 n \\
n\end{array}\right)$ & $\frac{3 n-4}{n}\left(\begin{array}{c}2 n-3 \\
n-1\end{array}\right)$ & 418 & 2431 & 17342 \\
\hline
\end{tabular}

In order to prove that there is a bijection between positive noncrossing partitions and Hom-configurations in $\mathcal{C}(Q)$ we will need to use the braid group action on the set of exceptional sequences of a fixed length. This action can also be called mutations of exceptional sequences.

We will now recall the notion of this braid group action and some useful facts. For more details we refer the reader to [11].

Given an exceptional sequence $E$ in $K Q$-mod, let $C(E)$ denote the smallest full subcategory of $K Q$-mod which contains $E$ and is closed under extensions, kernels and cokernels.

Let $B_{r}$ be the braid group on $r$ strings, with generators $\sigma_{1}, \ldots, \sigma_{r-1}$ satisfying the braid relations $\sigma_{i} \sigma_{j}=\sigma_{j} \sigma_{i}$ if $|i-j| \geq 2$ and $\sigma_{i} \sigma_{i+1} \sigma_{i}=\sigma_{i+1} \sigma_{i} \sigma_{i+1}$.

Proposition 5.5 [11] The following holds:

(1) Given an exceptional sequence $E$ in $K Q-\bmod , C(E)$ is equivalent to $K Q^{\prime}$ - mod where $Q^{\prime}$ is a quiver with no oriented cycles and with the number of vertices given by the length of $E$.

(2) If $(X, Y)$ is an exceptional sequence in $K Q-\bmod$ then there are unique indecomposable modules $R_{Y} X, L_{X} Y$ such that $\left(Y, R_{Y} X\right),\left(L_{X} Y, X\right)$ are exceptional sequences in $C(X, Y)$.

(3) Let $E=\left(X_{1}, \ldots, X_{r}\right)$ be an exceptional sequence and $1 \leq i<r$. Then $\left(X_{1}, \ldots, X_{i-1}, X_{i+1}, Y, X_{i+2}, \ldots, X_{r}\right)$ is an exceptional sequence in $C(E)$ if and only if $Y \simeq R_{X_{i+1}} X_{i}$.

Analogously, $\left(X_{1}, \ldots, X_{i-1}, Z, X_{i}, X_{i+2}, \ldots, X_{r}\right)$ is an exceptional sequence in $C(E)$ if and only if $Z \simeq L_{X_{i}} X_{i+1}$. 
(4) The braid group $B_{r}$ acts on the set of exceptional sequences of length $r$ by

$$
\begin{gathered}
\sigma_{i}\left(X_{1}, \ldots, X_{r}\right)=\left(X_{1}, \ldots, X_{i-1}, X_{i+1}, R_{X_{i+1}} X_{i}, X_{i+2}, \ldots, X_{r}\right) \\
\sigma_{i}^{-1}\left(X_{1}, \ldots, X_{r}\right)=\left(X_{1}, \ldots, X_{i-1}, L_{X_{i}} X_{i+1}, X_{i}, X_{i+2}, \ldots, X_{r}\right) .
\end{gathered}
$$

(5) The braid group action preserves the product of the corresponding reflections in the Weyl group.

(6) The braid group $B_{n}$ on $n$ strings acts transitively on the set of complete exceptional sequences.

The notion of exceptional sequences is related to Weyl group theory via the following theorem.

Theorem 5.6 [17, 18] Let c be a Coxeter element adapted to $Q$ (with respect to sinks, as above). Given a set of $n$ positive roots $\beta_{1}, \ldots, \beta_{n}$, the sequence $\left(M_{\beta_{1}}, \ldots, M_{\beta_{n}}\right)$ of modules associated to the positive roots (by Gabriel's Theorem), is an exceptional sequence if and only if $t_{\beta_{1}} \ldots t_{\beta_{n}}=c$.

We note that the implication from left to right in Theorem 5.6 follows from Proposition 5.5(5).

We are now able to prove the following theorem.

Theorem 5.7 There is a bijection between the positive noncrossing partitions and the sincere Hom-free sets in $K Q$-mod.

Proof Let the map $\varphi$ from $N C^{+}\left(W_{Q}\right)$ to the set of sincere Hom-free sets in $K Q$ - mod be defined as follows. Given a positive noncrossing partition $u$ with absolute length $r$, there is a $T$-reduced expression for $c$ which has a $T$-reduced expression $t_{\beta_{1}} \ldots t_{\beta_{r}}$ for $u$ as a prefix. By Theorem 5.6, the indecomposable modules corresponding to the reflections in this $T$-reduced expression for $c$ give rise to a complete exceptional sequence, and so in particular, $E=\left(E_{1}, \ldots, E_{r}\right)$, where $E_{i}$ denotes the indecomposable module associated to $t_{\beta_{i}}$, is an exceptional sequence. By Proposition 5.5(1) $C(E)$ is equivalent to $K Q^{\prime}$ - $\bmod$ where $Q^{\prime}$ is a quiver with $r$ vertices and no oriented cycles. We define $\varphi(u)$ to be the set of simple objects $S^{\prime}:=\left\{S_{1}^{\prime}, \ldots, S_{r}^{\prime}\right\}$ in $C(E)$.

Obviously, $S^{\prime}$ is a Hom-free set in $K Q$-mod. Suppose, for a contradiction, that $S^{\prime}$ is not sincere. Observe that the support of $C(E)$, i.e., the support of the modules in $C(E)$, is the same as the support of $E$. Hence, $E$ is not sincere either. But then $u=$ $t_{\beta_{1}} \ldots t_{\beta_{r}}$ would lie in the parabolic subgroup generated by the simple roots appearing in the $\beta_{i}$, for $1 \leq i \leq r$, when they are written as linear combinations of the simple roots. This subgroup is a proper parabolic subgroup since $E$ is not sincere, which contradicts the fact that $u$ is a positive noncrossing partition. Hence $\varphi(u)$ is indeed a sincere Hom-free set in $K Q$ - mod.

In order to check that this map is well defined we recall some results from [18]. In this paper the authors give a bijection, called cox, between the set of finitely generated wide subcategories of $K Q-\bmod$ and $N C\left(W_{Q}\right)$. A wide subcategory is, by definition, an exact Abelian subcategory closed under extensions. Any finitely generated 
wide subcategory $\mathcal{A}$ of $K Q$-mod is of the form $\mathcal{A}=K Q^{\prime}$-mod, where $Q^{\prime}$ is a finite acyclic quiver (cf. [18, Corollary 2.22]). Given a finitely generated wide subcategory $\mathcal{A}, \operatorname{cox}(\mathcal{A})$ is defined to be $t_{S_{1}^{\prime}} \ldots t_{S_{k}^{\prime}}$, where $\left(S_{1}^{\prime}, \ldots, S_{k}^{\prime}\right)$ are the simple objects of $\mathcal{A}$, ordered into an exceptional sequence. By $[18, \operatorname{Lemma} 3.10], \operatorname{cox}(\mathcal{A})=t_{F_{1}} \ldots t_{F_{r}}$, for any exceptional sequence $\left(F_{1}, \ldots, F_{r}\right)$ in $\mathcal{A}$.

Let $t_{\gamma_{1}} \ldots t_{\gamma_{r}}$ be another $T$-reduced expression for $u$, and $E^{\prime}=\left(E_{1}^{\prime}, \ldots, E_{r}^{\prime}\right)$ be the corresponding exceptional sequence. Note that $C(E)$ and $C\left(E^{\prime}\right)$ are finitely generated wide subcategories of $K Q$-mod. We have $\operatorname{cox}(C(E))=t_{\beta_{1}} \ldots t_{\beta_{r}}=u=t_{\gamma_{1}} \ldots t_{\gamma_{r}}=$ $\operatorname{cox}\left(C\left(E^{\prime}\right)\right)$. Because cox is an injective map, we have $C(E)=C\left(E^{\prime}\right)$ and so $\varphi$ is well defined.

In order to prove that $\varphi$ is an injective map, let $u, v \in N C^{+}(W)$ be such that $\varphi(u)=\varphi(v)=S^{\prime}$. Then, in particular, $u$ and $v$ must have the same absolute length, say $r$. Let $u=t_{\beta_{1}} \ldots t_{\beta_{r}}$ and $v=t_{\gamma_{1}} \ldots t_{\gamma_{r}}$ be $T$-reduced expressions. Let $E$ and $E^{\prime}$ be the corresponding exceptional sequences of $u$ and $v$, respectively. We know $S^{\prime}$ can be ordered into an exceptional sequence in $K Q$ - mod, so let us now view $S^{\prime}$ as such a sequence rather than just a set of modules. Due to the transitive action of the braid group $B_{r}$ in $C(E), S^{\prime}$ can be obtained from $E$ by a sequence of mutations in $C(E)$. Analogously, $E^{\prime}$ can be obtained from $S^{\prime}$ by a sequence of mutations in $C\left(E^{\prime}\right)$. Note that all of these mutations can be seen as mutations in $K Q$-mod. So we have a sequence of mutations in $K Q$-mod taking $E^{\prime}$ to $E$. It follows then by Proposition 5.5(5) that $u=t_{\beta_{1}} \ldots t_{\beta_{r}}=t_{\gamma_{1}} \ldots t_{\gamma_{r}}=v$, as we wanted.

To prove that $\varphi$ is surjective let $\mathcal{T}$ be a sincere Hom-free set in $K Q$-mod and $\mathcal{T} \sqcup \mathcal{U}$ be the corresponding Hom-configuration (4.4). If we order the elements of $\mathcal{T} \sqcup \mathcal{U}$ with respect to the total order $\leq$, we obtain an exceptional sequence where the first $k$ terms are the modules (as $\leq$ satisfies (1)), using Proposition 3.6. Assume $\left(X_{1}, \ldots, X_{k}, X_{k+1}, \ldots, X_{n}\right)$ is this ordering. Then $\left(X_{1}, \ldots, X_{k}, \bar{X}_{k+1}, \ldots, \bar{X}_{n}\right)$ is an exceptional sequence in $K Q$ - mod, by Lemma 3.2. By Theorem 5.6, we have

$$
c=t_{X_{1}} \ldots t_{X_{k}} t_{\bar{X}_{k+1}} \ldots t_{\bar{X}_{n}} .
$$

Let $u=t_{X_{1}} \ldots t_{X_{k}}$. By (4), $u$ is a noncrossing partition. Suppose $u$ is not positive. Then $u \in W_{S \backslash\{s\}}$, for some simple reflection $s$. By Lemma 5.3, $t_{X_{i}} \in W_{S \backslash\{s\}}$, for all $1 \leq i \leq k$. This means that $\mathcal{T}$ does not have support at the vertex associated to the simple reflection $s$, which contradicts the hypothesis. Hence $u$ is a positive noncrossing partition.

Since $\mathcal{T}$ is a Hom-free set with $k$ elements, it follows from Lemma 4.2 that $\mathcal{T}$ is the set of simple objects of $C(\mathcal{T})$, so $\varphi(u)=\mathcal{T}$, and we are done.

Corollary 5.8 The number of Hom-configurations in $\mathcal{C}(Q)$ is given by the positive Fuss-Catalan number.

To summarize, we have a bijection $\rho$ between $N C^{+}\left(W_{Q}\right)$ and the set of Homconfigurations in $\mathcal{C}(Q)$ (regarded as lying in $\mathcal{E}(Q)$ ), given by $\rho=\beta \varphi$, where $\varphi$ is defined in Theorem 5.7 and $\beta$ is defined in Theorem 4.8.

We will study the relation between the bijection $\rho$ and the bijection $\theta$ between 1-noncrossing partitions and $\mathrm{Hom}_{\leq 0}$-configurations (contained in $K Q$ - $\bmod \cup$ $K Q-\bmod [1])$, given by A. Buan, I. Reiten and H. Thomas in [10, Theorem 7.3]. 
Definition 5.9 A 1-noncrossing partition associated to $W_{Q}$ is a pair $\left(u_{1}, u_{2}\right)$ of elements of $W_{Q}$ such that

$$
c=u_{1} u_{2} \quad \text { and } \quad l_{T}\left(u_{1}\right)+l_{T}\left(u_{2}\right)=n .
$$

The pair $\left(u_{1}, u_{2}\right)$ is said to be a positive 1-noncrossing partition if $u_{1}$ is not contained in any proper parabolic subgroup. We denote by $N C_{1}\left(W_{Q}\right)$ (respectively, $N C_{1}^{+}\left(W_{Q}\right)$ ) the set of 1-noncrossing partitions (respectively, positive 1-noncrossing partitions) associated to $W_{Q}$.

Note that the map $n c$ sending $\left(u_{1}, u_{2}\right)$ to $u_{1}$ is a bijection between $N C_{1}\left(W_{Q}\right)$ and $N C\left(W_{Q}\right)$. Moreover, the restriction of $n c$ to $N C_{1}^{+}\left(W_{Q}\right)$ is a bijection between this set and $N C^{+}\left(W_{Q}\right)$.

The bijection $\rho$ can be described as follows:

Remark 5.10 Let $\left(u_{1}, u_{2}\right) \in N C_{1}^{+}\left(W_{Q}\right)$, and let $t_{1} \cdots t_{r}$ and $t_{r+1} \cdots t_{n}$ be $T$-reduced expressions for $u_{1}$ and $u_{2}$ respectively. Let $\mathcal{E}_{1}=\left(E_{1}, \ldots, E_{r}\right)$ and $\mathcal{E}_{2}=\left(E_{r+1}, \ldots, E_{n}\right)$ be the corresponding exceptional sequences in $K Q$-mod. Let $\mathcal{S}_{i}$ be the set of simple objects in $C\left(\mathcal{E}_{i}\right)$, for $i=1,2$.

Then $\rho\left(u_{1}\right)=\mathcal{S}_{1} \cup \mathcal{S}_{2}[1]$.

Proof We have $\rho\left(u_{1}\right)=\mathcal{S}_{1} \cup U[1]$, where $U$ is the set of simple objects in $\mathcal{S}_{1}^{\perp}$. If we prove that $\mathcal{S}_{1}^{\perp}=C\left(\mathcal{E}_{2}\right)$ then we are done.

Note that $\left(\mathcal{E}_{1}, \mathcal{E}_{2}\right)$ is a complete exceptional sequence in $K Q$-mod. Also, $\mathcal{S}_{1}$ can be ordered into an exceptional sequence and this sequence can be obtained from $\mathcal{E}_{1}$ by a sequence of mutations due to the transitive action of the braid group in $C\left(\mathcal{E}_{1}\right)$. These mutations can be seen as mutations in $K Q$-mod, so that $\left(\mathcal{S}_{1}, \mathcal{E}_{2}\right)$ is a complete exceptional sequence in $K Q$-mod. This implies that $\mathcal{S}_{1}$ is a complete exceptional sequence for ${ }^{\perp} \mathcal{E}_{2}$. Hence, by [11, Lemma 4(b)], it follows that $C\left(\mathcal{E}_{2}\right)=\mathcal{S}_{1}^{\perp}$, as we wanted to prove.

The bijection $\theta$ from $N C_{1}\left(W_{Q}\right)$ to the set of $\mathrm{Hom}_{\leq 0}$-configurations contained in $K Q-\bmod \cup K Q-\bmod [1]$, in [10, Theorem 7.3] (for $m=1$ ), has a similar description to the one in Remark 5.10. Before describing $\theta$ explicitly, we note that the Coxeter element $c^{\prime}$ used in [10] is adapted to $Q$ with respect to sources, i.e., $c^{\prime}=c^{-1}$. Also, the notion of exceptional sequence in [10] is the conventional one, i.e., $\left(E_{1}, \ldots, E_{r}\right)$ is an exceptional sequence (in the conventional sense) if

$$
\operatorname{Ext}_{K Q}\left(E_{j}, E_{i}\right)=0=\operatorname{Hom}_{K Q}\left(E_{j}, E_{i}\right),
$$

for $j>i$.

We will now describe $\theta$. Let $\left(u_{1}, u_{2}\right) \in N C_{1}\left(W_{Q}\right)$. Pick an exceptional sequence (in the conventional sense) $\left(E_{1}, \ldots, E_{n}\right)$ such that the first $l_{T}\left(u_{1}\right)$ terms (respectively, last $l_{T}\left(u_{2}\right)$ terms) correspond to a factorization of $u_{1}$ (respectively, $u_{2}$ ) into reflections. Let $\mathcal{E}_{i}$ denote the exceptional sequence corresponding to $u_{i}$ and $F_{i}$ denote the set of simples of $C\left(\mathcal{E}_{i}\right)$, for $i=1,2$. Then $\theta\left(u_{1}, u_{2}\right)=F_{2} \cup F_{1}[1]$. 
The inverse of $\theta$ is described as follows (for more details see the proof of [10, Theorem 7.3]): Let $\mathcal{X}$ be a $\operatorname{Hom}_{\leq 0}$-configuration in $\mathcal{D}^{b}(Q)$ and order it into an exceptional sequence (in the conventional sense) in such a way that the objects in $K Q-\bmod [1]$ appear before than those in $K Q$ - mod. Then $\theta^{-1}(\mathcal{X})=\left(u_{1}, u_{2}\right)$, where $u_{1}$ (respectively, $u_{2}$ ) is obtained by taking the product of the summands of $\mathcal{X}$ in $K Q$ - $\bmod [1]$ (respectively, $K Q$-mod), respecting the order of the exceptional sequence.

Recall the 1-1 correspondence $\mu$ between Hom-configurations in $\mathcal{C}(Q)$ (lying in $\mathcal{E}(Q))$ and $\mathrm{Hom}_{\leq 0}$-configurations contained in $\mathcal{D}_{\leq 1}^{(\geq 0)-}$ given in Remark 3.8. The relationship between the bijections $\rho$ and $\phi$ is given in the following proposition.

Proposition 5.11 Let $\mathcal{T}$ be a Hom-configuration in $\mathcal{C}(Q)$, regarded as lying in $\mathcal{E}(Q)$. Then $n c \circ \theta^{-1}(\mu(\mathcal{T}))=\left(\rho^{-1}(\mathcal{T})\right)^{-1}$. Hence, in particular, the set of noncrossing partitions corresponding under the bijection $\theta$ to $\mathrm{Hom}_{\leq 0}$-configurations contained in $\mathcal{D}_{\leq 1}^{(\geq 0)-}$ is in fact $N C_{1}^{+}\left(W_{Q}\right)$.

Proof Let

$$
\mathcal{T}=\left\{X_{1}, \ldots, X_{r}, X_{r+1}[1], \ldots, X_{n}[1]\right\}
$$

be a Hom-configuration in $\mathcal{C}(Q)$, whose objects lie in $\mathcal{E}(Q)$ and are ordered into an exceptional sequence with respect to the total order $\leq$.

Then

$$
\mu(\mathcal{T})=\left\{\tau^{-1} X_{r+1}, \ldots, \tau^{-1} X_{n}, X_{1}[1], \ldots, X_{r}[1]\right\}
$$

is a $\mathrm{Hom}_{\leq 0}$-configuration contained in $\mathcal{D}_{\leq 1}^{(\geq 0)-}$, and the elements are ordered into an exceptional sequence with respect to the total order $\leq$.

The corresponding exceptional sequence in the conventional sense (as in [10]) is $\left(X_{r}[1], \ldots, X_{1}[1], \tau^{-1} X_{n}, \ldots, \tau^{-1} X_{r+1}\right)$. Hence we have

$$
\theta^{-1}(\mu(\mathcal{T}))=\left(t_{r} \cdots t_{1}, t_{\tau^{-1} X_{n}} \cdots t_{\tau^{-1} X_{r+1}}\right) .
$$

On the other hand, $\rho^{-1}(\mathcal{T})=t_{1} \cdots t_{r}=\left(n c \circ \theta^{-1}(\mu(\mathcal{T}))\right)^{-1}$, and the proposition follows.

\section{Riedtmann combinatorial configurations}

In this section we give a link between Hom-configurations in $\mathcal{C}(Q)$ and the notion of configurations introduced by Riedtmann.

Definition 6.1 A set $\mathcal{T}$ of isomorphism classes of indecomposable objects of $\mathcal{D}^{b}(Q)$ is called a (Riedtmann) combinatorial configuration if it satisfies the following properties:

(1) $\operatorname{Hom}(X, Y)=0$ for all $X, Y$ in $\mathcal{T}, X \neq Y$,

(2) For all $Z \in$ ind $\mathcal{D}^{b}(Q)$, there exists $X \in \mathcal{T}$ such that $\operatorname{Hom}(Z, X) \neq 0$. 
A combinatorial configuration $\mathcal{T}$ is said to be $\tau$ [2]-periodic (or just periodic) if for every object $X$ in $\mathcal{T}$, we have $\tau^{k} X[2 k] \in \mathcal{T}$ for all $k \in \mathbb{Z}$.

Riedtmann proved that these combinatorial configurations are $\tau$ [2]-periodic in the cases when $Q$ is of type $A$ or $D$ (cf. [22, 23]).

We will only consider periodic combinatorial configurations and our aim is to prove that they are in bijection with Hom-configurations in $\mathcal{C}(Q)$.

Lemma 6.2 If $\mathcal{T}$ is a periodic combinatorial configuration, then the restriction of $\mathcal{T}$ to $\mathcal{E}(Q)$, viewed as a set of objects in $\mathcal{C}(Q)$, is a Hom-configuration in $\mathcal{C}(Q)$.

Proof Let us denote the restriction of $\mathcal{T}$ to $\mathcal{E}(Q)$ by $\mathcal{T}^{\prime}$. It follows from property 1 of Definition 6.1 and from the periodicity of $\mathcal{T}$ that $\mathcal{T}^{\prime}$ is a Hom-free set in $\mathcal{C}(Q)$. The maximality follows also from the fact that $\mathcal{T}$ is periodic, since this means that every object $Y$ in $\mathcal{T}$ is of the form $Y=\tau^{i} Y^{\prime}[2 i]$ for some $i \in \mathbb{Z}$ and some object $Y^{\prime} \in \mathcal{T}^{\prime}$, and from property 2 of Definition 6.1 .

Using Lemma 6.2 and the fact that the number of Hom-configurations in $\mathcal{C}(Q)$ is given by the positive Fuss-Catalan number, it is enough to show that the number of periodic combinatorial configurations is also given by the positive Fuss-Catalan number to get the bijection between these two notions of configurations.

In order to check this, we use some results presented in [6]. Namely the authors introduce another notion of configuration, which we shall refer to as BLR-configuration. Such configurations are periodic (cf. [6, Proposition 1.1]), and they are a subset of the set of periodic combinatorial configurations (for more details see the introduction in [6]).

Theorem 6.3 There is a bijection between the following objects:

(1) BLR-configurations in $\mathcal{D}^{b}(Q)$;

(2) periodic combinatorial configurations in $\mathcal{D}^{b}(Q)$;

(3) Hom-configurations in $\mathcal{C}(Q)$.

Remark 6.4 We have just seen that the following hold:

$\{$ BLR-configurations $\} \subseteq\{$ periodic combinatorial configurations $\}$

$$
\hookrightarrow\{\text { Hom-configurations in } \mathcal{C}(Q)\} .
$$

Hence, the only thing we need to check to prove Theorem 6.3 is that the number of BLR-configurations is given by the positive Fuss-Catalan number, i.e., the number of Hom-configurations in $\mathcal{C}(Q)$, by Corollary 5.8 .

If $Q$ is a quiver of type $A$, Bretscher, Läser and Riedtmann prove that BLRconfigurations in $\mathcal{D}^{b}(Q)$ are in bijection with pedigrees with $n$ vertices (cf. main theorem in introduction and Sect. 6.2 in [6]). By definition, a pedigree is a subtree of 
the oriented tree:
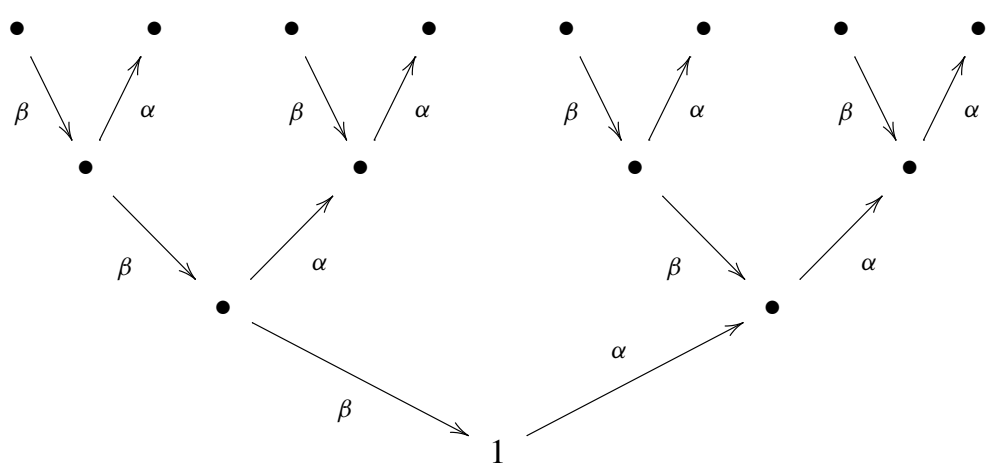

which contains the lowest vertex 1 .

Pedigrees with $n$ vertices are in 1-1 correspondence with binary trees. We recall that a binary tree is a rooted tree (trees are drawn growing upwards, by convention) in which each vertex $i$ has at most two children, i.e., vertices adjacent to $i$ which are above it in the tree. Each child of a vertex is designated as its left or right child.

The correspondence is described as follows: the lowest vertex 1 corresponds to the root, and $x$ is a right (left) child of $y$ if and only if we have $y \stackrel{\alpha}{\longrightarrow} x(x \stackrel{\beta}{\longrightarrow} y)$ in the pedigree.

It is known that the number of binary trees with $n$ vertices is given by $\frac{1}{n+1}\left(\begin{array}{c}2 n \\ n\end{array}\right)$, which is the positive Fuss-Catalan number $C^{+}\left(A_{n}\right)$. So we are done in type $A$.

It is proved in [6] (see end of Sect. 7.5) that the number of BLR-configurations in type $D_{n}$ is also given by the corresponding positive Fuss-Catalan number. So it remains to check type $E$.

In [6], the authors define two partitions of BLR-configurations into classes, the isomorphism classes and the equivalence classes. In order to define isomorphisms and equivalences of BLR-configurations, we need to define reflection in a horizontal line for type $E_{6}$ and reflection in a vertical line for types $E_{6}, E_{7}$ and $E_{8}$.

In type $E_{6}$, whose Dynkin diagram is given by

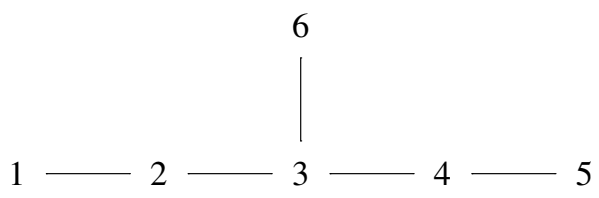

the nontrivial diagram automorphism $\alpha$ of the Dynkin diagram is given by $\alpha(x)=$ $(6-x) \bmod 6$, for $x=1, \ldots, 6$. Here we use modular arithmetic using the repre- 
sentatives $\{1, \ldots, 6\}$. This diagram automorphism induces an automorphism of the translation quiver $\mathbb{Z} E_{6}$, which shall be called reflection in a horizontal line.

Now for types $E_{6}, E_{7}$ and $E_{8}$, fix an alternating orientation of the corresponding Dynkin diagrams and take a copy of the resulting quiver $\Delta$ in the translation quivers $\mathbb{Z} E$, where $E$ is $E_{6}, E_{7}$ or $E_{8}$. The line connecting the sources is called a vertical line and the corresponding reflection is the automorphism $\phi$ of the underlying unoriented graph of $\mathbb{Z} E$ which is defined by induction as follows: $\phi(x)=x$, for $x$ a point of $\mathbb{Z} E$ in the vertical line, and given $x \rightarrow y$ in $\mathbb{Z} E$ with $\phi(x)$ already defined, define $\phi(y)$ as the unique vertex of $\mathbb{Z} E$ such that $\phi(y) \rightarrow \phi(x)$ is an arrow in $\mathbb{Z} E$ and $\phi(y)$ belongs to the $\tau$-orbit of $y$. There is a second kind of vertical line, which is the line connecting the sinks of the copy of $\Delta$ in $\mathbb{Z} E$. The corresponding automorphism of the underlying unoriented graph of $\mathbb{Z} E$ is defined similarly.

Isomorphisms of BLR-configurations come from automorphisms of the translation quiver, which are given by $\tau^{k}$, with $k \in \mathbb{Z}$, or by reflection in a horizontal line in type $E_{6}$. Two BLR-configurations are said to be equivalent if they are isomorphic or one is isomorphic to the reflection of the other in a vertical line.

In types $E_{7}$ and $E_{8}$ there is no reflection in a horizontal line. Hence, each isomorphism class has $h-1$ elements: a representative $\mathcal{T}$ and $\tau^{k}(\mathcal{T})$, with $1 \leq k \leq h-2$, as $\tau^{h-1}(\mathcal{T})=\mathcal{T}$. Hence the number of BLR-configurations is given by multiplying the number of isomorphism classes with $h-1$. In [6] the authors state that there are 143 and 598 isomorphism classes for type $E_{7}$ and $E_{8}$ respectively. Since $h-1$ equals 17 for type $E_{7}$ and 29 for type $E_{8}$, the number of BLR-configurations is 2431 and 17342 for type $E_{7}$ and $E_{8}$ respectively, which is the positive Fuss-Catalan number, as we wanted.

For type $E_{6}$ there are 17 equivalence classes. The authors of [6] list a representative for each of these equivalence classes. One can see that 12 of these equivalence classes are invariant under reflection in a vertical line. Thus there are $12+2 \times 5=22$ isomorphism classes. One can easily check that 6 of these isomorphism classes are invariant under reflection in a horizontal line. Therefore, there are $6+16 \times 2=38$ BLRconfigurations up to $\tau$-translation. Hence there are $38 \times(h-1)=38 \times 11=418$ BLR-configurations in total, which is the positive Fuss-Catalan number for type $E_{6}$.

\section{Riedtmann's bijection for type $A$}

The notion of classical noncrossing partitions of $\{1, \ldots, n\}=[n]$ was introduced by Kreweras [20] in 1972 and it is defined as follows.

Definition 7.1 [20] A classical noncrossing partition of $[n]$ is a partition $\mathcal{P}=$ $\left\{\mathcal{B}_{1}, \ldots, \mathcal{B}_{m}\right\}$ of the set $\{1, \ldots, n\}$, where we call $\mathcal{B}_{i}$ a block of $\mathcal{P}$ for $1 \leq i \leq m$, with the property that if $1 \leq a<b<c<d \leq n$, with $a, c \in \mathcal{B}_{i}$ and $b, d \in \mathcal{B}_{j}$, then $\mathcal{B}_{i}=\mathcal{B}_{j}$.

One can interpret this as being a partition of the vertices of a regular $n$-gon, whose vertices are ordered clockwise from 1 to $n$, such that the convex hulls of its blocks are disjoint from each other. 
The set of classical noncrossing partitions of $[n]$ form a poset under refinement of partitions, and we denote this poset by $N C(n)$. It was proved by Biane that $N C(n)$ and $N C\left(A_{n-1}\right)$ are isomorphic posets:

Theorem 7.2 [3, Theorem 1] Given a permutation $\pi$ of $[n]$ write it as a product of disjoint cycles (including 1-cycles) and let $\{\pi\}$ denote the partition of $[n]$ given by these cycles. The map $\pi \mapsto\{\pi\}$ is a poset isomorphism between $N C\left(A_{n-1}\right)$ and $N C(n)$.

In this section $Q$ denotes the quiver of type $A_{n}$ with linear orientation:

$$
Q: n \longrightarrow n-1 \longrightarrow \cdots \longrightarrow 1 \text {. }
$$

Riedtmann [22] proved that there is a bijection between the set of combinatorial configurations of $\mathcal{D}^{b}(Q)$ and $N C(n)$. In order to describe this map we need the following notation.

We know there is a bijection between the AR-quiver $\Gamma\left(\mathcal{D}^{b}(Q)\right)$ of $\mathcal{D}^{b}(Q)$ and the stable translation quiver $\mathbb{Z} Q^{o p}$, which is defined as follows:

(1) Vertices: $\left(\mathbb{Z} Q^{o p}\right)_{0}:=\mathbb{Z} \times Q_{0}^{o p}$.

(2) Arrows: for vertices $(x, a),(y, b)$ of $\mathbb{Z} Q^{o p}, \quad(x, a) \longrightarrow(y, b)$ is an arrow in $\mathbb{Z} Q^{o p}$ if $x=y$ and $a \longrightarrow b$ is an arrow in $Q^{o p}$ or $y=x+1$ and $b \longrightarrow a$ is an arrow in $Q^{o p}$.

This bijection can be chosen so that the indecomposable projective $P_{i}$ corresponds to $(1, i)$, for $i \in[n]$. Observe that the indecomposable $K Q$-modules are the objects of $\mathbb{Z} Q^{o p}$ written in the form $(i, j)$ with $2 \leq i+j \leq n+1$, with $i \geq 1$.

Recall that combinatorial configurations of type $A$ are periodic and so by Lemma 6.2 they can be regarded as Hom-configurations in $\mathcal{C}(Q)$. Moreover, it was seen in Sect. 6 that the map in Lemma 6.2, which is the restriction of a combinatorial configuration to the fundamental domain $\mathcal{E}(Q)$, is in fact a bijection. The composition of this bijection with Riedtmann's map (cf. [22, 2.6]) can be described as follows.

Theorem 7.3 $[22,2.6]$ Let $\mathcal{P}=\left\{\mathcal{B}_{1}, \ldots, \mathcal{B}_{m}\right\}$ be a classical noncrossing partition of the vertices of a regular $n$-gon, and assume the elements of each $\mathcal{B}_{i}$ are in numerical order. Given $k \in[n]$, let $\mathcal{B}=\left\{k_{1}, \ldots, k_{s}\right\}$ be the block that contains $k$. So $k=k_{r}$ for some $1 \leq r \leq s$. Let $\psi\left(k_{r}\right):=\left(k_{(r+1)} \bmod s-k_{r}\right) \bmod n$. Here we use modular arithmetic using the representatives $\{1,2, \ldots, l\}$ when working $\bmod l$.

Then the set $\{(i, \psi(i)) \mid i \in[n]\}$ is a Hom-configuration in $\mathcal{C}(Q)$ and the map defined this way, which we will call $\gamma$, is a bijection between $N C(n)$ and the set of Hom-configurations in $\mathcal{C}(Q)$.

Example 7.4 Consider the noncrossing partition $\mathcal{P}=\{\{1,3\},\{2\},\{4\}\}$ of $\{1,2,3,4\}$. Then the image under $\gamma$ is $\{(1,2),(2,4),(3,2),(4,4)\}=\{12,1[1], 34,3[1]\}$, with the notation we introduced in Example 4.5 (but note that the quiver we are using here has linear orientation). 
Our aim is to check that the composition of the bijections in Theorems 5.7 and 4.8 gives a generalization of this result.

First, we will give a combinatorial description for the positive noncrossing partitions of type $A$.

Proposition 7.5 A classical noncrossing partition of $[n+1]$ is positive if and only if the vertices 1 and $n+1$ lie in the same block.

Proof Let $\mathcal{P}=\left\{\mathcal{B}_{1}, \ldots, \mathcal{B}_{m}\right\}$ be a classical noncrossing partition of $[n+1]$ and $u$ be the corresponding noncrossing partition of type $A_{n}$.

Suppose 1 and $n+1$ do not lie in the same block. Let $\mathcal{B}_{1}$ be the block which contains the vertex 1 , and write $\mathcal{B}_{1}=\left\{1, k_{2}, \ldots, k_{s}\right\}$ in numerical order. By assumption, $k_{s} \neq n+1$. This block corresponds to the cycle $\left(1 k_{2} \ldots k_{s}\right)$, which can be written as a product of reflections (i.e., transpositions in this case):

$$
\begin{aligned}
\left(1 k_{2} \ldots k_{s}\right) & =\left(1 k_{2}\right)\left(k_{2} k_{3}\right) \ldots\left(k_{s-1} k_{s}\right) \\
& =t_{\alpha_{1}+\cdots+\alpha_{k_{2}-1}} t_{\alpha_{k_{2}}+\cdots+\alpha_{k_{3}-1}} \cdots t_{\alpha_{k_{s-1}}+\cdots+\alpha_{k_{s}-1}} .
\end{aligned}
$$

This element belongs to the parabolic subgroup $W_{1}$ generated by the simple reflections $s_{\alpha_{1}}, s_{\alpha_{2}}, \ldots, s_{\alpha_{k_{s}}-1}$. Note that this subgroup is proper as $s_{\alpha_{k_{s}}} \notin W_{1}$.

Since $\mathcal{P}$ is noncrossing, there are no vertices $l$ and $m$ lying in the same block with $l<k_{s}<m$. Hence, the cycle $c_{i}$ corresponding to the block $\mathcal{B}_{i}$ lies in a parabolic subgroup $W_{i}$ which does not contain $s_{\alpha_{k_{s}}}$, and so $u=\prod_{i=1}^{m} c_{i}$ belongs to the product of the parabolic subgroups $W_{i}(1 \leq i \leq m)$, which is a proper parabolic subgroup. Hence the noncrossing partition $u$ is not positive.

Now suppose 1 and $n+1$ are in the same block, say $\mathcal{B}_{1}$. We have

$$
\mathcal{B}_{1}=\left\{1, k_{1,2}, \ldots, k_{1, s-1}, n+1\right\}
$$

with $1<k_{1,2}<\cdots<k_{1, s-1}<n+1$. The corresponding cycle $c_{1}=\left(1 k_{1,2} \ldots\right.$ $\left.k_{1, s-1} n+1\right)$ can be written in the form

$$
c_{1}=t_{\alpha_{1}+\cdots+\alpha_{k_{1,2}-1}} t_{\alpha_{k_{1,2}}+\cdots+\alpha_{k_{1,3}-1}} \cdots t_{\alpha_{k_{1, s-1}}+\cdots+\alpha_{n}} .
$$

Note that $l_{T}\left(c_{1}\right)=s-1$, since $s$ is the length of the cycle (cf. [4, Proposition 2.3]). Hence the product of reflections above is a $T$-reduced expression for $c_{1}$. Moreover, if $c_{i}$ is the cycle corresponding to $\mathcal{B}_{i}$, we have an expression for $u$ as a product of disjoint cycles, $u=\prod_{i=1}^{m} c_{i}$, and so $l_{T}(u)=\sum_{i=1}^{m} l_{T}\left(c_{i}\right)$ (cf. [4, Lemma 2.2]).

Let $E$ be the exceptional sequence in $K Q$ - $\bmod$ associated to this $T$-reduced expression of $u$, by Theorem 5.6. Due to the $T$-reduced expression for $c_{1}$, we see that $E$ has support on every vertex of $Q$, i.e., $E$ is sincere.

Consider $C(E) \simeq K Q^{\prime}$-mod, where the number of vertices of $Q^{\prime}$ equals $r$, the number of terms in $E$ (cf. [11, Lemma 5]). Let $S^{\prime}=\left\{S_{1}^{\prime}, \ldots, S_{r}^{\prime}\right\}$ be the set of simple objects in $C(E)$, ordered into an exceptional sequence. Due to the transitive action of the set of mutations on complete exceptional sequences in $C(E), E$ can be obtained from $S^{\prime}$ by a sequence of mutations. Hence $u=t_{\underline{\operatorname{dim}} S_{1}^{\prime}} \ldots t_{\underline{\operatorname{dim}}} S_{r}^{\prime}$, by Proposition 5.5(5). 
On the other hand, $\operatorname{supp} S^{\prime}=\operatorname{supp} C(E)=\operatorname{supp}(E)$, which implies that $S^{\prime}$ is a sincere Hom-free set in $K Q$ - mod. By Theorem 5.7, $\varphi^{-1}\left(S^{\prime}\right)=t_{\text {dim }} S_{1}^{\prime} \ldots t_{\text {dim }} S_{r}^{\prime}$ is a positive noncrossing partition, i.e., $u \in N C^{+}\left(A_{n}\right)$, as we wanted.

The following proposition follows easily from Proposition 7.5.

Proposition 7.6 Given a classical noncrossing partition $\mathcal{P}=\left\{\mathcal{B}_{1}, \ldots, \mathcal{B}_{m}\right\}$ of $[n]$, where $1 \in \mathcal{B}_{1}$, let $f(\mathcal{P})$ be the partition $\left\{\mathcal{B}_{1}^{\prime}, \ldots, \mathcal{B}_{m}^{\prime}\right\}$ of $[n+1]$ defined by

$$
\mathcal{B}_{i}^{\prime}= \begin{cases}\mathcal{B}_{1} \cup\{n+1\} & \text { if } i=1 \\ \mathcal{B}_{i} & \text { if } i \neq 1\end{cases}
$$

Then $f(\mathcal{P})$ is a positive noncrossing partition and $f: N C(n) \rightarrow N C^{+}(n+1)$, where $N C^{+}(n+1)$ is the image of $\mathrm{NC}^{+}\left(A_{n}\right)$ under the isomorphism between $\mathrm{NC}\left(A_{n}\right)$ and $N C(n+1)$, is a bijection.

Theorem 7.7 Let $\rho: \mathrm{NC}^{+}\left(A_{n}\right) \rightarrow\{$ Hom-configurations in $\mathcal{C}(Q)\}$ be the composition of the bijection $\varphi$ in Theorem 5.7 followed by the bijection $\beta$ in Theorem 4.8. Let $\gamma$ be Riedtmann's bijection (see Theorem 7.3). Then we have $\rho^{-1} \circ \gamma=f$.

Proof We recall that here we are using the notation for the stable translation quiver $\mathbb{Z} Q^{o p}$. Observe that the element $(i, j) \in \mathbb{Z} Q^{o p}$ with $i \geq 1$ and $i+j \leq n+1$ corresponds to the indecomposable module $M_{i j}$ whose dimension vector is given by

$$
\left(\underline{\operatorname{dim}} M_{i j}\right)_{l}= \begin{cases}1 & \text { if } l \in\{i, i+1, \ldots, i+j-1\} \\ 0 & \text { otherwise. }\end{cases}
$$

Note also that this indecomposable module corresponds to the transposition $(i i+j)=t_{\alpha_{i}+\cdots+\alpha_{i+j-1}}$.

Let $\mathcal{P}=\left\{\mathcal{B}_{1}, \ldots, \mathcal{B}_{m}\right\} \in N C(n)$. Let $\leq$ be the refinement to a total order of the partial order $\preceq$ which was fixed in the beginning of this paper. Then the elements of the Hom-configuration $\gamma(\mathcal{P})$ ordered with respect to this refinement form an exceptional sequence where the modules are the first elements. We can assume this refinement satisfies the following property: if the indecomposable objects $(i, \psi(i))$ and $(j, \psi(j))$ have the same degree, then $(i, \psi(i)) \leq(j, \psi(j))$ if $i<j$. This means that the modules in $\gamma(\mathcal{P})$ are ordered into an exceptional sequence in the following way:

$$
M_{i_{1}, \psi\left(i_{1}\right)}, \quad \ldots, \quad M_{i_{k}, \psi\left(i_{k}\right)},
$$

where $1 \leq i_{1}<\cdots<i_{k}$ and $i_{j}+\psi\left(i_{j}\right) \leq n+1$. So we have

$$
\rho^{-1}(\gamma(\mathcal{P}))=t_{\underline{\operatorname{dim}} M_{i_{1}, \psi\left(i_{1}\right)}} \cdots t_{\underline{\operatorname{dim}} M_{i_{k}, \psi\left(i_{k}\right)}} .
$$

Note that if $(i, \psi(i)),(j, \psi(j)) \in \gamma(\mathcal{P}) \cap K Q$ - $\bmod$ are such that $i$ and $j$ belong to different blocks, then the corresponding reflections commute. 
Hence we can write

$$
\rho^{-1}(\gamma(\mathcal{P}))=\prod_{j=1}^{m} \prod_{\substack{i \in \mathcal{B}_{j} \\ i+\psi(i) \leq n+1}} t_{\operatorname{dim}} M_{i \psi(i)},
$$

where the product corresponding to each block $\mathcal{B}_{j}$ respects the order in (5).

Given this, consider the block $\mathcal{B}_{1}=\left\{k_{11}=1, k_{12}, \ldots, k_{1, r_{1}-1}, k_{1 r_{1}}\right\}$ of $\mathcal{P}$. This block gives rise to the following elements in $\gamma(\mathcal{P})$ :

$\left(1, k_{12}-1\right), \quad\left(k_{12}, k_{13}-k_{12}\right), \quad \ldots, \quad\left(k_{1, r_{1}-1}, k_{1 r_{1}}-k_{1, r_{1}-1}\right), \quad\left(k_{1 r_{1}}, n-k_{1 r_{1}}+1\right)$.

We denote this set of elements by $\mathcal{T}_{1}$.

The corresponding indecomposable objects lie in $K Q$-mod, since they are of the form $(i, j)$ with $i \geq 1$ and $i+j \leq n+1$.

The reflections associated to the elements of $\mathcal{T}_{1}$ are:

$$
\left(1 k_{12}\right), \quad\left(k_{12} k_{13}\right), \quad \ldots, \quad\left(k_{1 r_{1}} n+1\right),
$$

respectively.

The part of the product in (6) corresponding to $\mathcal{B}_{1}$ is the following product:

$$
\begin{aligned}
\prod_{i \in \mathcal{B}_{1}} \underline{t_{\operatorname{dim}} M_{i, \psi(i)}} & =\left(1 k_{12}\right)\left(k_{12} k_{13}\right) \ldots\left(k_{1 r_{1}} n+1\right) \\
& =\left(1 k_{12} k_{13} \ldots k_{1 r_{1}} n+1\right) .
\end{aligned}
$$

Let $\mathcal{B}_{j}$ be any other block of $\mathcal{P}$, and write $\mathcal{B}_{j}=\left\{k_{j 1}, k_{j 2}, \ldots, k_{j r_{j}}\right\}$, with $k_{j 1}<$ $k_{j 2}<\cdots<k_{j r_{j}}$. Following the same argument as before, this block gives rise to the following objects in $\gamma(\mathcal{P})$ :

$$
\begin{aligned}
& \left(k_{j 1}, k_{j 2}-k_{j 1}\right), \quad\left(k_{j 2}, k_{j 3}-k_{j 2}\right), \quad \ldots, \quad\left(k_{j, r_{j}-1}, k_{j r_{j}}-k_{j, r_{j}-1}\right), \\
& \left(k_{j r_{j}}, n-k_{j r_{j}}+k_{j 1}\right) .
\end{aligned}
$$

All these objects but the last one lie in $K Q$ - $\bmod \left(\right.$ note that $\left(k_{j r_{j}}, n-k_{j r_{j}}+k_{j 1}\right) \notin$ $K Q-\bmod$ since $k_{j r_{j}}+\left(n-k_{j r_{j}}+k_{j 1}\right)=n+k_{j 1} \geq n+2$ as $\left.k_{j 1} \neq 1\right)$.

We have

$$
\begin{aligned}
\prod_{\substack{i \in \mathcal{B}_{j} \\
i(i) \leq n+1}} t_{\mathrm{dim}} M_{i, \psi(i)} & =\left(k_{j 1} k_{j 2}\right)\left(k_{j 2} k_{j 3}\right) \ldots\left(k_{j, r_{j}-1} k_{j r_{j}}\right) \\
& =\left(k_{j 1} k_{j 2} \ldots k_{j, r_{j}-1} k_{j r_{j}}\right) .
\end{aligned}
$$

Hence the blocks of $\rho^{-1}(\gamma(\mathcal{P}))$ are $\left\{k_{j 1}, \ldots, k_{j r_{j}}\right\}=\mathcal{B}_{j}$, with $2 \leq j \leq m$, and $\left\{1, k_{12}, \ldots, k_{1 r_{1}}, n+1\right\}=\mathcal{B}_{1} \cup\{n+1\}$, which allow us to conclude that $\rho^{-1} \circ \gamma=f$, as we wanted. 


\section{Mutations of Hom-configurations}

In this section we give a definition of mutation of Hom-configurations in $\mathcal{C}(Q)$, which will rely on Proposition 2.3. The first thing we need to do is to generalize, in the obvious way, this result.

Corollary 8.1 Let $\left\{X_{1}, \ldots, X_{k}\right\}$ be a Hom-free set in ind $\mathcal{C}(Q)$. Then

$$
{ }^{\perp}\left\{X_{1}, \ldots, X_{k}\right\}^{\perp}=\left\{Y \in \mathcal{C}(Q) \mid \operatorname{Hom}_{\mathcal{C}(Q)}\left(X_{i}, Y\right)=0=\operatorname{Hom}_{\mathcal{C}(Q)}\left(Y, X_{i}\right), \forall i \in[k]\right\}
$$

is equivalent to $\mathcal{C}\left(Q^{\prime}\right)$ where $Q^{\prime}$ is a disjoint union of quivers of Dynkin type and whose number of vertices is $n-k$.

Proof We prove this by induction on $k$. The case when $k=1$ is Proposition 2.3. Let $\left\{X_{1}, \ldots, X_{k}, X_{k+1}\right\}$ be a Hom-free set in $\mathcal{C}(Q)$. Then so is $\left\{X_{1}, \ldots, X_{k}\right\}$ and by induction we have

$$
{ }^{\perp}\left\{X_{1}, \ldots, X_{k}\right\}^{\perp} \simeq \mathcal{C}\left(Q^{\prime}\right),
$$

where $Q^{\prime}$ is a disjoint union of quivers of Dynkin type, whose sum of vertices is $n-k$, in other words, $Q^{\prime}=\bigsqcup_{i=1}^{t} Q^{i}$, where each $Q^{i}$ is a Dynkin quiver and $\sum_{i=1}^{t}\left|Q_{0}^{i}\right|=$ $n-k$.

We have $X_{k+1} \in{ }^{\perp}\left\{X_{1}, \ldots, X_{k}\right\}_{Q}^{\perp}$, and so by (7), there exists a unique $i \in[t]$ for which $X_{k+1} \in \mathcal{C}\left(Q^{i}\right)$. Given $Y \in \mathcal{C}\left(Q^{j}\right)$, with $j \neq i$, we have

$$
\operatorname{Hom}_{\mathcal{C}(Q)}\left(X_{k+1}, Y\right)=0=\operatorname{Hom}_{\mathcal{C}(Q)}\left(Y, X_{k+1}\right)
$$

since $\mathcal{C}\left(Q^{l}\right), l \in[t]$, are pairwise orthogonal. Hence $\bigsqcup_{j \neq i} \mathcal{C}\left(Q^{j}\right) \subseteq{ }^{\perp}\left(X_{k+1}\right)_{Q}^{\perp}$. Therefore,

$$
\begin{aligned}
{ }^{\perp}\left\{X_{1}, \ldots, X_{k+1}\right\}_{Q}^{\perp} & ={ }^{\perp}\left(X_{k+1}\right)_{Q}^{\perp} \cap{ }^{\perp}\left\{X_{1}, \ldots, X_{k}\right\}_{Q}^{\perp} \\
& \simeq{ }^{\perp}\left(X_{k+1}\right)_{Q}^{\perp} \cap\left(\bigsqcup_{l \in[t]} \mathcal{C}\left(Q^{l}\right)\right) \quad(\text { by }(7)) \\
& =\left({ }^{\perp}\left(X_{k+1}\right) \stackrel{\perp}{Q} \cap \mathcal{C}\left(Q^{i}\right)\right) \sqcup\left({ }^{\perp}\left(X_{k+1}\right)_{Q}^{\perp} \cap \bigsqcup_{j \neq i} \mathcal{C}\left(Q^{j}\right)\right) \\
& =\left({ }^{\perp}\left(X_{k+1}\right)_{Q}^{\perp} \cap \mathcal{C}\left(Q^{i}\right)\right) \sqcup\left(\bigsqcup_{j \neq i} \mathcal{C}\left(Q^{j}\right)\right) .
\end{aligned}
$$

Note that $\left.{ }^{\perp}\left(X_{k+1}\right) \stackrel{\perp}{Q} \cap \mathcal{C}\left(Q^{i}\right)\right) \simeq{ }^{\perp}\left(X_{k+1}\right)_{Q^{i}}^{\perp}$, which, by Proposition 2.3, is equivalent to $\mathcal{C}\left(Q^{\prime \prime}\right)$, where $Q^{\prime \prime}$ is a disjoint union of quivers of Dynkin type whose sum of vertices is $\left|Q_{0}^{i}\right|-1$, and we are done.

In representation theory, mutations are operations that act on a certain class of objects, and construct a new one from a given one by replacing a summand. For example, mutations of exceptional sequences and cluster mutations are of this type. 
In this case, however, this will not make much sense. Indeed, let $\mathcal{T}=\left\{X_{1}, \ldots, X_{n}\right\}$ be a Hom-configuration in $\mathcal{C}(Q)$, and suppose we remove one object $X_{i}$. Then, by Corollary 8.1, ${ }^{\perp}\left(\mathcal{T} \backslash X_{i}\right)_{Q}^{\perp}$ is equivalent to $\mathcal{C}\left(Q^{\prime}\right)$ where $Q^{\prime}$ is of type $A_{1}$. Hence, the only completion of $\mathcal{T}$, i.e., the only object $Y$ of $\mathcal{C}(Q)$ for which $\mathcal{T} \cup Y$ is a Homconfiguration is $X_{i}$. Therefore, in order to define mutations of Hom-configurations, we need to remove more than one object.

A particular case of Corollary 8.1, which will be useful later, is the following:

Corollary 8.2 Let $\left\{X_{1}, \ldots, X_{n}\right\}$ be a Hom-configuration in $\mathcal{C}(Q)$. If we remove two objects, say $X_{i}$, and $X_{j}$, with $i \neq j$, then ${ }^{\perp}\left\{X_{k} \mid k \neq i, j\right\}_{Q}^{\perp} \simeq \mathcal{C}\left(Q^{\prime}\right)$, where $Q^{\prime}$ is a quiver with two vertices which are either connected by a single arrow, i.e., $Q^{\prime}$ is of type $A_{2}$, or they are disconnected, i.e., $Q^{\prime}$ is of type $A_{1} \times A_{1}$.

Lemma 8.3 Let $Q^{\prime}$ be a quiver of type $A_{2}$. Then the only two Hom-configurations in $\mathcal{C}\left(Q^{\prime}\right)$ are the set of simple modules, and $\left\{P, P^{\prime}[1]\right\}$, where $P$ is the injectiveprojective $K Q^{\prime}$-module and $P^{\prime}$ is the simple projective $K Q^{\prime}$-module.

Given these results, we are able to give a definition of mutation of Homconfigurations in $\mathcal{C}(Q)$.

Definition 8.4 Let $i, j \in[n], i<j$. The mutation $\mu_{i, j}$ of the Hom-configuration $\mathcal{T}:=\left\{X_{1}, \ldots, X_{n}\right\}$ in $\mathcal{C}(Q)$ is defined as follows:

(1) If ${ }^{\perp}\left\{\mathcal{T} \backslash\left\{X_{i}, X_{j}\right\}\right\}_{Q}^{\perp}$ is equivalent to $\mathcal{C}\left(Q^{\prime}\right)$ where $Q^{\prime}$ is a quiver of type $A_{2}$, then $\mu_{i, j}(\mathcal{T})$ is the Hom-configuration obtained from $\mathcal{T}$ by replacing the Hom-configuration $\left\{X_{i}, X_{j}\right\}$ in $\mathcal{C}\left(Q^{\prime}\right)$ by the other possible Hom-configuration $\left\{X_{i}^{\prime}, X_{j}^{\prime}\right\}$ in this category (cf. Lemma 8.3).

(2) For the remaining case, i.e., if ${ }^{\perp}\left\{\mathcal{T} \backslash\left\{X_{i}, X_{j}\right\}\right\}_{Q}^{\perp}$ is equivalent to $\mathcal{C}\left(Q^{\prime}\right)$ where $Q^{\prime}$ is of type $A_{1} \times A_{1}$, then $\mu_{i, j}(\mathcal{T})=\mathcal{T}$.

Example 8.5 Consider the quiver $Q$ of type $A_{3}: 3 \longrightarrow 2 \longrightarrow 1$. The following figure shows the graph of mutations of the Hom-configurations in $\mathcal{C}(Q)$, where the vertices correspond to Hom-configurations and the edges correspond to mutations:

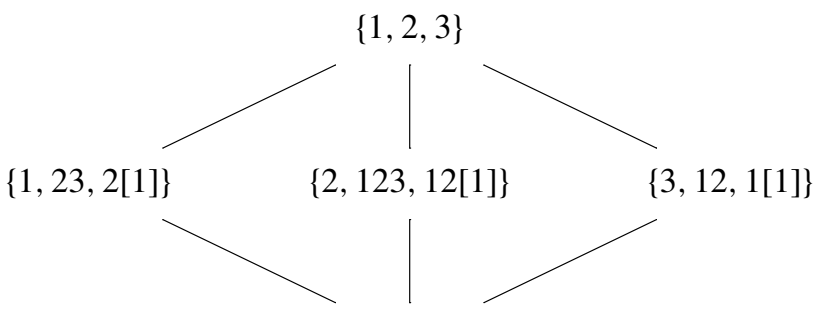

$\{123,1[1], 2[1]\}$

Proposition 8.6 The graph of mutations $\mathcal{G}(Q)$ of Hom-configurations in $\mathcal{C}(Q)$ is connected. 
Proof We prove this by induction on the number of vertices $n$. For $n=1$ there is nothing to prove.

It is easy to check that this proposition holds in the cases when $Q$ is of type $A_{2}$, $A_{3}$ and $D_{4}$.

Let now $Q$ be any other Dynkin quiver with $n$ vertices. Note that given a vertex $i$ of $Q$ there exists a vertex $j$ which is not a neighbor of $i$, i.e., there is no arrow between $i$ and $j$. First we claim that two Hom-configurations $\mathcal{T}$ and $\mathcal{T}^{\prime}$ in $\mathcal{C}(Q)$ with a common object $X$ are connected by a path in $\mathcal{G}(Q)$. Let $F$ be the equivalence between ${ }^{\perp} X^{\perp}$ and $\mathcal{C}\left(Q^{\prime}\right)$, where $Q^{\prime}$ is a Dynkin quiver with $n-1$ vertices (see Proposition 2.3). Note that $F(\mathcal{T} \backslash X)$ and $F\left(\mathcal{T}^{\prime} \backslash X\right)$ are Hom-configurations in $\mathcal{C}\left(Q^{\prime}\right)$. By induction $\mathcal{G}\left(Q^{\prime}\right)$ is connected, so $F(\mathcal{T} \backslash X)$ and $F\left(\mathcal{T}^{\prime} \backslash X\right)$ are connected by a path in $\mathcal{G}\left(Q^{\prime}\right)$, i.e., there is a sequence of mutations $\mu_{1}, \ldots, \mu_{k}$ such that $\mu_{1} \ldots \mu_{k}(F(\mathcal{T} \backslash X))=$ $F\left(\mathcal{T}^{\prime} \backslash X\right)$. This sequence of mutations can be lifted to a sequence of mutations in $\mathcal{C}(Q)$ fixing the object $X$. Hence $\mathcal{T}$ and $\mathcal{T}^{\prime}$ are connected by a path in $\mathcal{G}(Q)$, as we wanted to prove.

Now fix a Hom-configuration $\mathcal{T}$ in $\mathcal{C}(Q)$. We want to prove that $\mathcal{T}$ is connected to the simple Hom-configuration $\mathcal{S}$ by a path in $\mathcal{G}(Q)$. By Lemma 4.2(2) there exists a $K Q$-module $X$ in $\mathcal{T}$, and there is a sequence of reflection functors $R=R_{i_{k}} \ldots R_{i_{1}}$, where $i_{j}$ is a sink in $\sigma_{i_{j-1}} \ldots \sigma_{i_{1}}(Q)$, such that $R_{i_{k}} \ldots R_{i_{1}}(X)$ is a simple $K \sigma_{R}(Q)$-module. Since $R(\mathcal{T})$ is a Hom-configuration in $\mathcal{C}\left(\sigma_{R}(Q)\right)$ and it contains a simple module, it follows from what we claimed above that $R(\mathcal{T})$ and the set of simple $K \sigma_{R}(Q)$-modules $\mathcal{S}_{\sigma_{R}(Q)}$ are connected by a path in $\mathcal{G}\left(\sigma_{R}(Q)\right)$. Note that $i_{k}$ is a source in $\sigma_{R}(Q)$ and by assumption, there is a vertex $j$ which is not a neighbor of $i_{k}$. Hence $R_{i_{k}}^{-}\left(S_{\sigma_{R}(Q)}(j)\right)=S_{\sigma_{i_{k}} \sigma_{R}(Q)}(j)$ (cf. [1, VII.5.4]). Therefore $R_{i_{k}}^{-}\left(\mathcal{S}_{\sigma_{R}(Q)}\right)$ is connected to $\mathcal{S}_{\sigma_{i_{k}} \sigma_{R}(Q)}$ by a path in $\mathcal{G}\left(\sigma_{i_{k}} \sigma_{R}(Q)\right)$ since it contains a simple $K \sigma_{i_{k}} \sigma_{R}(Q)$-module. Since $R_{i_{k}}^{-} R(\mathcal{T})$ and $R_{i_{k}}^{-}\left(\mathcal{S}_{\sigma_{R}(Q)}\right)$ are connected by a path in $\mathcal{G}\left(\sigma_{i_{k}} \sigma_{R}(Q)\right)$, it follows that $R_{i_{k}}^{-} R(\mathcal{T})$ is also connected to $\mathcal{S}_{\sigma_{i_{k}} \sigma_{R}(Q)}$ by a path in $\mathcal{G}\left(\sigma_{i_{k}} \sigma_{R}(Q)\right)$. Using the same argument when we apply the reflections $R_{i_{k-1}}^{-} \ldots R_{i_{1}}^{-}$, we deduce that $\mathcal{T}$ is connected to $\mathcal{S}_{Q}$ by a path in $\mathcal{G}(Q)$.

Acknowledgements The author would like to express her gratitude to her supervisor, Robert Marsh, for his help and advice, and also to Aslak Buan, Idun Reiten and Hugh Thomas for making available a preliminary version of their paper [10], and to Aslak Buan for helpful conversations. She would also like to thank the anonymous referee for useful comments and suggestions and to Fundacão para a Ciência e Tecnologia, for their financial support through Grant SFRH/ BD/ 35812/ 2007.

\section{References}

1. Assem, I., Simson, D., Skowroński, A.: Elements of the Representation Theory of Associative Algebras, vol 1. Techniques of Representation Theory. London Mathematical Society Student Texts, vol. 65. Cambridge University Press, Cambridge (2006)

2. Bessis, D.: The dual braid monoid. Ann. Sci. Éc. Norm. Super. 36, 647-683 (2003)

3. Biane, P.: Some properties of crossings and partitions. Discrete Math. 175, 41-53 (1997)

4. Brady, T.: A partial order on the symmetric group and new $K(\pi, 1)$ 's for the braid group. Adv. Math. 161(1), 20-40 (2001)

5. Brady, T., Watt, C.: Noncrossing partition lattices in finite real reflection groups. Trans. Am. Math. Soc. 360, 1983-2005 (2008) 
6. Bretscher, O., Läser, C., Riedtmann, C.: Selfinjective and simply connected algebras. Manuscr. Math. 36(3), 253-307 (1982)

7. Brüning, K.: Thick subcategories of the derived category of a hereditary algebra. Homol. Homot. Appl. 9(2), 165-176 (2007)

8. Buan, A.B., Marsh, R.J., Reineke, M., Reiten, I., Todorov, G.: Tilting theory and cluster combinatorics. Adv. Math. 204, 572-618 (2006)

9. Buan, A.B., Reiten, I., Thomas, H.: $m$-noncrossing partitions and $m$-clusters. DMTCS proc. (subm.), extended abstract

10. Buan, A., Reiten, I., Thomas, H.: From $m$-clusters to $m$-noncrossing partitions via exceptional sequences. Preprint arXiv:1007.0928v1 [math.RT]

11. Crawley-Boevey, W.: Exceptional sequences of representations of quivers. In: Dlab, V., Lenzing, H. (eds.) Representations of Algebras, Proc. Ottawa 1992. Canadian Math. Soc. Conf. Proc, vol. 14, pp. 117-124. Amer. Math. Soc., Providence (1993)

12. Fomin, S., Zelevinsky, A.: Cluster algebras I: Foundations. J. Am. Math. Soc. 15(2), 497-529 (2002)

13. Fomin, S., Zelevinsky, A.: Cluster algebras II: Finite type classification. Invent. Math. 154(1), 63-121 (2003)

14. Fomin, S., Zelevinsky, A.: Y-systems and generalized associahedra. Ann. Math. (2) 158(3), 977-1018 (2003)

15. Gabriel, P.: Auslander-Reiten sequences and representation-finite algebras. In: Representation Theory, I, Proc. Workshop, Carleton Univ., Ottawa, Ont., 1979, pp. 1-71. Springer, Berlin (1980)

16. Happel, D.: Triangulated Categories in the Representation Theory of Finite-Dimensional Algebras. London Mathematical Society Lecture Note Series, vol. 119. Cambridge University Press, Cambridge (1988)

17. Igusa, K., Schiffler, R.: Exceptional sequences and clusters. J. Algebra 323(8), 2183-2202 (2010)

18. Ingalls, C., Thomas, H.: Noncrossing partitions and representations of quivers. Compos. Math. 145(6), 1533-1562 (2009)

19. Keller, B.: On triangulated orbit categories. Doc. Math. 10, 551-581 (2005)

20. Kreweras, G.: Sur les partitions non croisées d'un cycle. Discrete Math. 1, 333-350 (1972)

21. Reading, N.: Clusters, Coxeter-sortable elements and noncrossing partitions. Trans. Am. Math. Soc. 359, 5931-5958 (2007)

22. Riedtmann, C.: Representation-finite selfinjective algebras of class $A_{n}$. In: Representation Theory II, Prof. Second Internat. Conf., Carleton Univ., Ottawa, Ont. 1979. Lecture Notes in Math., vol. 832, pp. 449-520. Springer, Berlin (1980)

23. Riedtmann, C.: Configurations of $\mathbb{Z} D_{n}$. J. Algebra 82(2), 309-327 (1983)

24. Ringel, C.: The braid group action on the set of exceptional sequences of a hereditary Artin algebra. In: Abelian Group Theory and Related Topics, Oberwolfach, 1993. Contemp. Math., vol. 171, pp. 339-352. Amer. Math. Soc., Providence (1994)

25. Schofield, A.: Semi-invariants of quivers. J. Lond. Math. Soc. 43, 385-395 (1991) 